\title{
Computational Aspects of Group Extensions and Their Applications in Topology
}

\author{
Karel Dekimpe and Bettina Eick
}

\section{CONTENTS}

1. Introduction

2. Algorithms for Polycyclic Groups

3. Extensions with Certain Properties

4. Computing Betti Numbers

5. Almost Bieberbach Groups

Appendix A

References
2000 AMS Subject Classification: Primary 20-04, 20F16, 20F18; Secondary 57-04, 57M05

Keywords: Almost crystallographic groups, algorithms for polycyclic groups, torsion-free extensions, Betti numbers
We describe algorithms to determine extensions of infinite polycyclic groups having certain properties. In particular, we are interested in torsion-free extensions and extensions whose Fitting subgroup has a minimal centre. Then we apply these methods in topological applications. We discuss the calculation of Betti numbers for compact manifolds, and we describe algorithmic approaches in classifying almost Bieberbach groups.

\section{INTRODUCTION}

The aims of this paper are two-fold. First, we describe algorithms to compute certain extensions of polycyclic groups. Then we apply these methods in solving a variety of topological problems.

Methods to determine the first and second cohomology group of a polycyclic group are described in [Eick 01b]. As the second cohomology group corresponds to the equivalence classes of extensions, these methods yield descriptions for all extensions of a polycyclic group. Here we consider the case of a polycyclic group acting on the infinite cyclic group, and we extend these algorithms to check the existence of extensions with certain properties. In particular, we are interested in torsion-free extensions and extensions whose Fitting subgroups have a minimal centre. We develop practical methods to solve these problems; implementations of our methods are available in the ACLIB package [Dekimpe and Eick 01a] of GAP.

Then, we consider Eilenberg-MacLane manifolds of type $K(G, 1)$ for polycyclic groups $G$. Many topological invariants of such spaces correspond to algebraic invariants of $G$. In particular, the (co)homology of a $K(G, 1)$ manifold is completely determined by the (co)homology of $G$. As applications of the algorithms introduced, we present methods for:

- Computing Betti numbers of a closed $K(G, 1)$ manifold. If the dimension of $K(G, 1)$ is at most 6 , then we can determine all Betti numbers of $K(G, 1)$;

(c) A K Peters, Ltd. 1058-6458/2001 \$0.50 per page Experimental Mathematics 11:2, page 183 
- Constructing the almost Bieberbach groups of small dimension as initiated in [Dekimpe 96]. These groups are the fundamental groups of the infranilmanifolds;

- Investigating infra-nilmanifolds modeled on Heisenberg Lie groups. We use these methods to extend the results obtained in [Lee and Szczepański 00].

\section{ALGORITHMS FOR POLYCYCLIC GROUPS}

A group $G$ is called polycyclic if it has a polycyclic series; that is, a subnormal series with cyclic factors. The number of infinite cyclic factors in a polycyclic series of a group $G$ is an invariant of $G$ called the Hirsch length $h(G)$.

Several practical algorithms for computations in polycyclic groups are known. For example, a basic setup of methods is described in [Sims 94, Chapter 9], and a more extended collection of algorithms has been introduced recently in [Eick 01b]. In particular, [Eick 01b] contains methods to determine torsion, to compute the Fitting subgroup and the centre, and to calculate the first and second cohomology group for a polycyclic group. Thus, using the cohomology groups, we also develop approaches to construct complements and extensions.

We want to apply and extend this collection of methods for our purposes. In this section, we briefly recall the main basic ideas of computations with polycyclic groups which we will need later. In particular, we introduce the fundamental concept of polycyclic presentations in Section 2.1 and their parameterized version in Section 2.2. Then we discuss the determination of cohomology groups and extensions in Section 2.3. Implementations of the algorithms described in this section are available as part of the POLYCYCLIC package [Eick and Nickel 00] of GAP.

\subsection{Polycyclic Presentations}

Let $\left(r_{1}, \ldots, r_{n}\right)$ be a sequence with $r_{i} \in \mathbb{N} \cup\{\infty\}$ for $\mathbb{N}=\{1,2, \ldots\}$, the natural numbers, and denote $I=\{i \in$ $\left.\{1, \ldots, n\} \mid r_{i}<\infty\right\}$, the finite index set for the sequence. A polycyclic presentation with exponents $\left(r_{1}, \ldots, r_{n}\right)$ is a presentation of the type

$$
\begin{gathered}
G=\left\langle g_{1}, \ldots, g_{n} \quad\right| g_{i}^{g_{j}}=w_{i, j}\left(g_{j+1}, \ldots, g_{n}\right) \\
\quad \text { for } 1 \leq j<i \leq n, \\
g_{i}^{g_{j}^{-1}}=w_{j, i}\left(g_{j+1}, \ldots, g_{n}\right) \\
\quad \text { for } 1 \leq j<i \leq n, j \notin I \\
g_{i}^{r_{i}}=w_{i, i}\left(g_{i+1}, \cdots, g_{n}\right) \\
\text { for } i \in I\rangle
\end{gathered}
$$

where $w_{i, j}$ is a word in the considered generators and their inverses. It is straightforward to observe that a group is polycyclic if and only if it has a polycyclic presentation.

Further, a polycyclic presentation reflects the polycyclic structure of the group it defines. In particular, its generators, $g_{1}, \ldots, g_{n}$, define a polycyclic series for $G$ with subgroups $G_{i}=\left\langle g_{i}, \ldots, g_{n}\right\rangle$. This feature can be used to design effective algorithms for polycyclic groups.

If $G$ is defined by a polycyclic presentation as above, then each element $g \in G$ can be represented by a collected word of the form $g=g_{1}^{e_{1}} \cdots g_{n}^{e_{n}}$ with $e_{i} \in \mathbb{Z}$ and $0 \leq e_{i}<r_{i}$ for $i \in I$. If an element $g \in G$ is given as an arbitrary word in the generators of $G$, then the "collection algorithm" can be used to determine a collected word defining the same element as $g$.

In summary, this algorithm takes an arbitrary word $g$ in the generators of $G$ and uses the relations of $G$ to substitute basic uncollected subwords of the type $g_{i}^{ \pm 1} g_{j}^{ \pm 1}$ for $j<i$ or $g_{i}^{e_{i}}$ for $i \in I$ and $e_{i} \notin\left\{0, \ldots, r_{i}-1\right\}$ by equivalent collected words. Iterating this substitution process, the algorithm eventually produces a collected word in the generators of $G$ which is equivalent to $g$.

It is possible that different collected words define the same element in $G$. If each element of $G$ is represented by a unique collected word, then we call the considered polycyclic presentation consistent.

It is not difficult to observe that each polycyclic group has a consistent polycyclic presentation. The consistency of a polycyclic presentation can be checked using the following lemma.

Lemma 2.1. A polycyclic presentation with generators $\left(g_{1}, \ldots, g_{n}\right)$ and exponents $\left(r_{1}, \ldots, r_{n}\right)$ is consistent if and only if the words on the righthand sides and the lefthand sides of the following equations yield the same collected word, where the subwords in brackets are collected first. ([Sims 94, page 424])

$$
\begin{array}{rll}
g_{k}\left(g_{j} g_{i}\right)=\left(g_{k} g_{j}\right) g_{i} & \text { for } & k>j>i \\
\left(g_{j}^{r_{j}}\right) g_{i}=g_{j}^{r_{j}-1}\left(g_{j} g_{i}\right) & \text { for } & j>i, j \in I \\
g_{j}\left(g_{i}^{r_{i}}\right)=\left(g_{j} g_{i}\right) g_{i}^{r_{i}-1} & \text { for } & j>i, i \in I \\
\left(g_{i}^{r_{i}}\right) g_{i}=g_{i}\left(g_{i}^{r_{i}}\right) & \text { for } & i \in I \\
g_{j}=\left(g_{j} g_{i}^{-1}\right) g_{i} & \text { for } & j>i, i \notin I
\end{array}
$$

In our later applications we usually have a consistent polycyclic presentation for the considered groups given. For example, for almost-crystallographic groups described by unipotent-by-finite rational matrix representations, such a presentation can be determined easily. 


\subsection{Parameterized Polycyclic Presentations}

In this section, we investigate polycyclic presentations in which the relations may incorporate certain indeterminates. We will show that such presentations can be used to describe extensions of polycyclic groups in a computationally useful form. We consider a group $F$ which is given by the consistent polycyclic presentation

$$
\begin{gathered}
F=\left\langle f_{1}, \ldots, f_{m}\right| f_{i}^{f_{j}}=v_{i, j}\left(f_{j+1}, \ldots, f_{n}\right) \\
\text { for } 1 \leq j<i \leq m, \\
f_{i}^{f_{j}^{-1}}=v_{j, i}\left(f_{j+1}, \ldots, f_{m}\right) \\
\text { for } 1 \leq j<i \leq m, j \notin I \\
f_{i}^{r_{i}}=v_{i, i}\left(f_{i+1}, \cdots, f_{m}\right) \\
\text { for } i \in I\rangle
\end{gathered}
$$

and an $F$-module $M$ of the form $M \cong \mathbb{Z}^{d}$. Each extension $G$ of $M$ by $F$ has a consistent polycyclic presentation on generators $g_{1}, \ldots, g_{m}, m_{1}, \ldots, m_{d}$ such that $M$ is generated by $m_{1}, \ldots, m_{d}$ (we identify $M$ with a subgroup of $G$ ) and $g_{i} M=f_{i}$ for $1 \leq i \leq m$. Suppose that the $F$-module structure of $M$ is given explicitly via $F \rightarrow G L(d, \mathbb{Z}): f_{i} \mapsto F_{i}$ and denote $F_{i}^{-1}=E_{i}$. Then we obtain a consistent polycyclic presentation of $G$ with relations of the following three types:

$$
\begin{aligned}
& \left(R_{1}\right) g_{i}^{g_{j}}=v_{i, j}\left(g_{j+1}, \ldots, g_{m}\right) t_{i, j}\left(m_{1}, \ldots, m_{d}\right) \\
& g_{i}^{g_{j}^{-1}}=v_{j, i}\left(g_{j+1}, \ldots, g_{m}\right) t_{j, i}\left(m_{1}, \ldots, m_{d}\right), \\
& (1 \leq j<i \leq m, j \notin I), \\
& g_{i}^{r_{i}}=v_{i, i}\left(g_{i+1}, \ldots, g_{m}\right) t_{i, i}\left(m_{1}, \ldots, m_{d}\right) \\
& (i \in I) \text {. }
\end{aligned}
$$

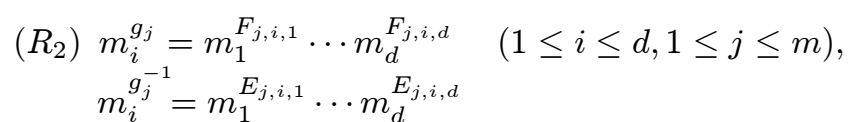

$$
\begin{aligned}
& (1 \leq i \leq d, 1 \leq j \leq m, j \notin I) . \\
& \left(R_{3}\right) m_{i}^{m_{j}^{ \pm 1}}=m_{i} \quad(1 \leq j<i \leq d)
\end{aligned}
$$

The relations of type $\left(R_{3}\right)$ are relations of $M$. The relations of type $\left(R_{2}\right)$ reflect the action of $G$ on $M$ and thus are determined by the $F$-module structure of $M$ only.

We study the relations of type $\left(R_{1}\right)$ in more detail. In general, as observed in Section 2.1, the righthand sides of these relations have the form $w_{i, j}\left(g_{j+1}, \ldots, g_{m}, m_{1}, \ldots, m_{d}\right)$. Since $M$ is an abelian normal subgroup of $G$, we can rewrite these righthand sides such that

$$
\begin{aligned}
& w_{i, j}\left(g_{j+1}, \ldots, g_{m}, m_{1}, \ldots, m_{d}\right)= \\
& v_{i, j}\left(g_{j+1}, \ldots, g_{m}\right) t_{i, j}\left(m_{1}, \ldots, m_{d}\right) .
\end{aligned}
$$

Then the relations of type $\left(R_{1}\right)$ exhibit the given polycyclic presentation of the factor group $F \cong G / M$. Further, the tails $t_{i, j}$ of these relations are words in the generators of $M$. Let $\mathcal{T}$ be the set of those index pairs $(i, j)$ such that $t_{i, j}$ is defined and denote $l=|\mathcal{T}| \leq m^{2}$. Since $M$ is free abelian, the tails are of the form

$$
t_{i, j}\left(m_{1}, \ldots, m_{d}\right)=m_{1}^{t_{i, j, 1}} \cdots m_{d}^{t_{i, j, d}} \quad \text { for }(i, j) \in \mathcal{T}
$$

and thus each tail $t_{i, j}$ can be identified with an element of $M$; this element is uniquely defined by the relations of $F$, the module structure of $M$ and the extension $G$. We denote $t=\left(t_{i, j} \mid(i, j) \in \mathcal{T}\right) \in M^{l}$ the tailvector consisting of all tails $t_{i, j}$. We observe that this tailvector is the only part in the above relations which depends on the extension $G$. In summary, we have observed the following lemma.

Lemma 2.2. If $G$ is an extension of the free abelian group $M$ by the polycyclic group $F$, and a consistent polycyclic presentation for $F$ as well as the explicit $F$ module structure for $M$ is given, then $G$ can be defined by a consistent polycyclic presentation $P(t)$ with generators $g_{1}, \ldots, g_{m}, m_{1}, \ldots, m_{d}$ and relations $R_{1}(t), R_{2}, R_{3}$, where the relations depend on the given presentation for $F$, the $F$-module structure for $M$, and the relations $R_{1}(t)$ additionally depend on a tailvector $t \in M^{l}$.

Let $T=\left(T_{i, j} \mid(i, j) \in \mathcal{T}\right)$ be a vector of $l$ different indeterminates which can take values in $M$. Then we can consider the parameterized polycyclic presentation $P(T)$ obtained as in Lemma 2.2. Each extension $G$ of $M$ by $F$ can be defined by a specialization $P(t)$ for some vector $t \in M^{l}$. Using the methods introduced in Section 2.1, we can compute with a polycyclic presentation $P(t)$ for each $t \in M^{l}$. In the following, we observe that certain restricted computations can be performed in $P(T)$ without specifying the values $t$ of $T$ in $M^{l}$.

In particular, we are interested in a collection algorithm for $P(T)$. As a preliminary example, we collect the word $\left(g_{m} g_{1}\right) g_{1}$ in $P(T)$ assuming that $1, m \notin I$ and using the relations righthand side $v_{m, 1}=g_{m}$ :

$$
\begin{aligned}
\left(g_{m} g_{1}\right) g_{1} & =\left(g_{1} g_{m}^{g_{1}}\right) g_{1}=\left(g_{1} v_{m, 1} T_{m, 1}\right) g_{1} \\
& =\left(g_{1} g_{m} T_{m, 1}\right) g_{1}=g_{1}^{2} g_{m}^{g_{1}} T_{m, 1}^{g_{1}} \\
& =g_{1}^{2}\left(v_{m, 1} T_{m, 1}\right) T_{m, 1}^{g_{1}}=g_{1}^{2}\left(g_{m} T_{m, 1}\right) T_{m, 1}^{g_{1}} \\
& =g_{1}^{2} g_{m} T_{m, 1}^{1+g_{1}} .
\end{aligned}
$$

Hence we obtain a similar collected form as in Section 2.1, but incorporating certain expressions in the 
indeterminates in $T$. To shorten notation, we denote $T=\left(T_{1}, \ldots, T_{l}\right)$ by taking an arbitrary ordering on the $T_{i, j}$, and we recall that each $T_{i}$ can take values in $M$ only. In particular, the indeterminates in $T$ commute with each other and with each element of $M$. Then we can consider a word in the generators of $P(T)$ as collected, if it is of the form

$$
g_{1}^{e_{1}} \cdots g_{m}^{e_{m}} \cdot m_{1}^{e_{m+1}} \cdots m_{d}^{e_{m+d}} \cdot T_{1}^{f_{1}} \cdots T_{l}^{f_{l}}
$$

where $e_{i} \in \mathbb{Z}$ for $1 \leq i \leq m+d$ with $0 \leq e_{i}<r_{i}$ if $r_{i}<\infty$ and $f_{j} \in \mathbb{Z} F$ for $1 \leq j \leq l$. With this notation for collected words, the collection algorithm of Section 2.1 generalizes readily as shown in the above example.

\subsection{Computing Low-Dimensional Cohomology Groups}

Let $F$ be a polycyclic group with an $F$-module $M$ where $F$ acts from the right. In this section, we describe or refer to methods for computing the cohomology groups $H^{i}(F, M)$ with $0 \leq i \leq 2$. Although the algorithms considered here apply to many types of modules $M$, we restrict the following description to the case when $M$ is free abelian of finite rank $d$, since this is the case needed in later applications. Thus the $F$-module structure of $M$ can be expressed using a homomorphism $\psi: F \rightarrow$ $G L(d, \mathbb{Z})$.

As usual, we define the $i$-th cohomology group as $H^{i}(F, M)=Z^{i}(F, M) / B^{i}(F, M)$. To compute cohomology groups, we need to determine the groups of cocycles $Z^{i}(F, M)$ and the groups of coboundaries $B^{i}(F, M)$ using a computationally useful representation for them.

2.3.1 Determining $H^{0}(F, M)$ and $H^{1}(F, M)$. The computation of $H^{0}(F, M)$ is straightforward: $B^{0}(F, M)=0$ and $Z^{0}(F, M)=C_{M}(F)$, the fixed points in $M$ under action of $F$. Thus $H^{0}(F, M)=Z^{0}(F, M)$ can be obtained from generators for the image of the action homomorphism $\psi$.

The construction of $H^{1}(F, M)$ is not difficult either. If $F=\left\langle f_{1}, \ldots, f_{m}\right\rangle$, then we can define $Z^{1}(F, M) \rightarrow$ $M^{n}: \delta \mapsto\left(\delta\left(f_{1}\right), \ldots, \delta\left(f_{m}\right)\right)$. This is a monomorphism of abelian groups whose image is a subgroup of $M^{m} \cong \mathbb{Z}^{d m}$. In [Eick 01a], there are methods described to compute bases for the images of $Z^{1}(F, M)$ and $B^{1}(F, M)$ under this embedding. These images can be used to compute the first cohomology group $H^{1}(F, M)$ explicitly.

2.3.2 Determining $H^{2}(F, M)$ and Extensions. The determination of $H^{2}(F, M)$ and its relationship to computing extensions is described in [Eick 01b]. Since we want to extend this method in later sections, we include a report on the construction of $Z^{2}(F, M)$ here. First, we recall the well-known correspondence between the second cohomology group and extensions in the following lemma. We refer to [Robinson 82] for more background on this topic.

Lemma 2.3. Let $F$ be a group with an $F$-module $M$.

(i) Each cocycle $\gamma \in Z^{2}(F, M)$ determines an extension $G_{\gamma}$ of $M$ by $F$. This extension can be written as $G_{\gamma}=\{(f, m) \mid f \in F, m \in M\}$ with multiplication $(f, m)(h, n)=\left(f h, \gamma(f, h) m^{h} n\right)$. Further, $M \cong\{(1, m) \mid m \in M\}$ and $G_{\gamma} \rightarrow F:(f, m) \mapsto f$ is an epimorphism.

(ii) Each extension $G$ of $M$ by $F$ determines a cocycle $\gamma \in Z^{2}(F, M)$ with respect to a transversal $t: F \rightarrow G$ via $\gamma(f, h)=t(f h)^{-1} t(f) t(h)$ for all $f, h \in F$.

Let $F$ be a polycyclic group which is defined by a consistent polycyclic presentation and let $M \cong \mathbb{Z}^{d}$ be an $F$-module. Then, as discussed in Section 2.2, each extension $G$ of $M$ by $F$ can be defined by a consistent polycyclic presentation $P(t)$ for some $t \in M^{l}$. Hence if a cocycle $\gamma \in Z^{2}(F, M)$ is given, then $\gamma$ determines an extension $G_{\gamma}$ of $M$ by $F$ and this, in turn, determines a vector $t \in M^{l}$. Note that $t \in M^{l}$ is defined uniquely by $\gamma$ and thus we obtain a map

$$
\phi: Z^{2}(F, M) \rightarrow M^{l}: \gamma \mapsto t .
$$

Lemma 2.4. The map $\phi$ is a homomorphism of abelian groups with $\operatorname{ker}(\phi) \leq B^{2}(F, M)$. Thus $H^{2}(F, M) \cong$ $\phi\left(Z^{2}(F, M)\right) / \phi\left(B^{2}(F, M)\right)$.

Proof: Let $\gamma_{1}, \gamma_{2} \in Z^{2}(F, M)$. Then $\left(\begin{array}{ll}\gamma_{1} & \gamma_{2}\end{array}\right)(f, h)=$ $\gamma_{1}(f, h) \gamma_{2}(f, h)$, and thus $G_{\gamma_{1} \gamma_{2}}$ has a polycyclic presentation of the type $P\left(t_{1} t_{2}\right)$ with $t_{i}=\phi\left(\gamma_{i}\right)$ for $i=1,2$. Thus $\phi\left(\gamma_{1} \gamma_{2}\right)=t_{1} t_{2}=\phi\left(\gamma_{1}\right) \phi\left(\gamma_{2}\right)$ and $\phi$ is a homomorphism. Now let $\gamma \in \operatorname{ker}(\phi)$. Then $t=\phi(\gamma)=0$. Thus $P(t)$ defines a split extension of $M$ by $F$. Hence, $\gamma \in B^{2}(F, M)$.

Our aim now is to determine a basis for the image of $\phi$ and hence to obtain a representation for $Z^{2}(F, M)$. For this purpose, we use the following lemma from [Eick 01b].

Lemma 2.5. Let $P(T)$ be a parameterized presentation arising from a consistent polycyclic presentation for a group $F$ and an $F$-module $M$. Then $t \in \phi\left(Z^{2}(F, M)\right)$ if and only if $P(t)$ is consistent. 
It remains to determine those elements $t \in M^{l}$ such that $P(t)$ is a consistent polycyclic presentation. For this purpose, we use the collection algorithm for $P(T)$ described in Section 2.2 to evaluate the relations of the consistency check in Lemma 2.1. For each of these consistency relations, we then obtain collected forms for their right- and lefthand side. These yield equations of the type

$$
g_{1}^{e_{1}} \cdots g_{m}^{e_{m}} T_{1}^{f_{1}} \cdots T_{l}^{f_{l}}=g_{1}^{e_{1}^{\prime}} \cdots g_{m}^{e_{m}^{\prime}} T_{1}^{f_{1}^{\prime}} \cdots T_{l}^{f_{l}^{\prime}}
$$

for $e_{i}, e_{i}^{\prime} \in \mathbb{Z}$ and $f_{j}, f_{j}^{\prime} \in \mathbb{Z} F$. Since $F$ is given by a consistent polycyclic presentation, $e_{i}=e_{i}^{\prime}$ for $1 \leq i \leq m$. Hence the equation can be rewritten

$$
T_{1}^{f_{1}-f_{1}^{\prime}} \cdots T_{l}^{f_{l}-f_{l}^{\prime}}=1
$$

The elements $t \in M^{l}$ for which $P(t)$ is consistent are exactly the solutions of these resulting equations for $T$. It remains to solve this system of equations over the free abelian group $M$. For this purpose, we identify $M \cong \mathbb{Z}^{d}$ and switch to additive notation. Using the explicit $F$ module structure of $M$, we can translate each coefficient $f_{i}-f_{i}^{\prime} \in \mathbb{Z} F$ to an integral matrix in $M_{d \times d}(\mathbb{Z})$. Thus the obtained system of equations translates into a homogeneous system of linear equations over $\mathbb{Z}$. This can be solved readily using a Hermite normal form algorithm; we obtain an explicit representation for $\phi\left(Z^{2}(F, M)\right) \leq M^{l}$.

Example 2.6. We consider the infinite dihedral group $F$ given by its consistent polycyclic presentation $\left\langle f_{1}, f_{2}\right.$ | $\left.f_{1}^{2}=1, f_{2}^{f_{1}}=f_{2}^{-1}\right\rangle$. This group has a module $M=\mathbb{Z}^{2}$ on which $F$ acts via $\psi: F \rightarrow G L(2, \mathbb{Z})$ defined by

$$
\psi\left(f_{1}\right)=\left(\begin{array}{rr}
-1 & 0 \\
0 & 1
\end{array}\right) \text { and } \psi\left(f_{2}\right)=\left(\begin{array}{ll}
1 & 1 \\
0 & 1
\end{array}\right) .
$$

The corresponding parameterized polycyclic presentation $P(T)$ has generators $g_{1}, g_{2}, g_{3}, g_{4}$ with $g_{1}=\left(f_{1}, 1\right), g_{2}=$ $\left(f_{2}, 1\right), g_{3}=\left(1, m_{1}\right)$ and $g_{4}=\left(1, m_{2}\right)$. The relations of $P(T)$ of type $\left(R_{1}\right)$ read $g_{1}^{2}=T_{1}$ and $g_{2}^{g_{1}}=g_{2}^{-1} T_{2}$.

We evaluate the consistency relations of Lemma 2.1 in $P(T)$. There are two nontrivial relations that need to be considered: $g_{1}\left(g_{1}^{2}\right)=\left(g_{1}^{2}\right) g_{1}$ and $g_{2}\left(g_{1}^{2}\right)=\left(g_{2} g_{1}\right) g_{1}$. We collect the right- and lefthand side of the first relation and we obtain

$$
g_{1}\left(g_{1}^{2}\right)=g_{1} T_{1} \text { and }\left(g_{1}^{2}\right) g_{1}=T_{1} g_{1}=g_{1} T_{1}^{g_{1}} .
$$

Thus we have to solve $T_{1}^{g_{1}-1}=1$. Similarly, we find for the second consistency relation that $T_{1}^{g_{2}-1} T_{2}^{g_{1}-g_{2}}=1$.
Now we switch to additive notation and have to determine the nullspace of

$$
A=\left(\begin{array}{cc}
g_{1}-1 & g_{2}-1 \\
0 & g_{1}-g_{2}
\end{array}\right) .
$$

To determine this nullspace explicitly, we use the explicit $F$-module structure for $M$; that is, we consider the homomorphism $\psi: F \rightarrow G L(2, \mathbb{Z})$ and extend it to $\psi: \mathbb{Z} F \rightarrow M_{2 \times 2}(\mathbb{Z})$ and then to matrices over $\mathbb{Z} F$. This can be used to derive an explicit representation for $A$ as

$$
A \mapsto \bar{A}=\left(\begin{array}{rr|rr}
-2 & 0 & 0 & 1 \\
0 & 0 & 0 & 0 \\
\hline 0 & 0 & -2 & -1 \\
0 & 0 & 0 & 0
\end{array}\right) .
$$

The nullspace of $\bar{A}$ can now be computed easily as $\operatorname{ker}(\bar{A})=\langle(0,1,0,0),(0,0,0,1)\rangle \leq \mathbb{Z}^{4}$. Using the natural homomorphism $\mathbb{Z}^{4} \cong M^{2}$, we get $\phi\left(Z^{2}(F, M)\right) \cong \operatorname{ker}(\bar{A})$.

Further, $\phi\left(B^{2}(F, M)\right)=\langle(0,2,0,0),(0,0,0,1)\rangle$ in this example. Thus, in summary, $H^{2}(G, M) \cong \mathbb{Z} / 2 \mathbb{Z}$.

Remark 2.7. To determine $\phi\left(B^{2}(F, M)\right)$, we recall that a cocycle $\gamma \in B^{2}(F, M)$ defines a split extension of $M$ by $F$. Thus with $t=\phi(\gamma)$, we determine that the group defined by $P(t)$ contains a complement $K$ to $M$. Such a complement $K$ is generated by $g_{1} n_{1}, \ldots, g_{m} n_{m}$ for certain $n_{1}, \ldots, n_{m} \in M$, and this generating set for $K$ fulfills the relations of $F$. The vectors $t$ which exhibit such a complement can be determined readily.

\section{EXTENSIONS WITH CERTAIN PROPERTIES}

In this section, we present algorithms to check if extensions of $\mathbb{Z}$ by a polycyclic group $F$ with certain properties exist. In particular, we are interested in extensions which are torsion-free or which fulfill a condition on their Fitting subgroup. These properties are inspired by the topological questions we deal with later in this paper.

Since $F$ is polycyclic, there exist only finitely many normal subgroups of index 2 in $F$. Hence there are only finitely many module actions that $F$ can induce on $\mathbb{Z}$. Thus, to find a certain extension of $\mathbb{Z}$ by $F$, we can consider each of these module actions successively and check if an extension of the desired type exists for a fixed module structure.

We can assume that we have an $F$-module $M \cong \mathbb{Z}$. To find an extension with a certain property, we search for an element of $H^{2}(F, M)$ which yields such an extension. Note that $H^{2}(F, M)$ may be infinite, and thus we cannot simply check the considered property for each of the extension classes of $M$ by $F$. 


\subsection{Torsion-Free Extensions}

Let $F$ be a polycyclic group with an $F$-module $M$ of the form $M \cong \mathbb{Z}$. If $F$ is torsion-free, then each extension of $M$ with $F$ is torsion-free. But if $F$ contains torsion, then at least the split extension of $M$ by $F$ will also contain torsion. Our aim in this section is to describe a method to check if there exists a torsion-free extension of $M$ by $F$.

The following lemma yields the basic underlying observation. It uses the fact that a polycyclic group has only finitely many conjugacy classes of finite subgroups.

Lemma 3.1. Let $G$ be an extension of $M \cong \mathbb{Z}$ by $F$ written as $G=\{(f, m) \mid f \in F, m \in M\}$. We identify $M$ with $\{(1, m) \mid m \in M\}$. Let $\left\{H_{1}, \ldots, H_{s}\right\}$ be a set of conjugacy class representatives for the subgroups of prime order in F.

(i) If there exists a finite, noncyclic subgroup $H \leq F$, then $G$ contains torsion.

(ii) If $C_{M}\left(H_{i}\right) \neq M$ for some $i \in\{1, \ldots, s\}$, then $G$ contains torsion.

(iii) Suppose that $C_{M}\left(H_{i}\right)=M$ for $1 \leq i \leq s$ and denote $H_{i}=\left\langle h_{i}\right\rangle$ with order $p_{i}$. Then $G$ contains torsion if and only if $\left(h_{i}, 1\right)^{p_{i}} \in M^{p_{i}}$ for some $i \in\{1, \ldots, s\}$.

Proof: As a first step, we investigate a torsion-free extension $G$ of $M$ by a finite group $H$. Let $L=C_{G}(M)$, where $M$ is embedded as a subgroup in $G$. Since $M \cong \mathbb{Z}, L$ has index at most 2 in $G$. Further, $L$ is a torsion-free central extension of $M$ by the finite group $L / M$. Thus $L^{\prime}$ is a finite group and therefore $L^{\prime}=1$. Hence $L$ is abelian and, since it is also torsion-free, $L \cong \mathbb{Z}$. If $[G: L]=2$, then $G$ acts nontrivially on $M$ and thus it acts nontrivially on $L$. Hence $G$ is isomorphic to the infinite dihedral group and thus contains torsion-a contradiction. Thus $G=L \cong \mathbb{Z}$ and $G$ is a central extension of $M$ by a cyclic group $H$. Parts (i) and (ii) follow directly from this preliminary observation.

To prove (iii), we obseerve that if $G$ contains torsion, then there exists an element $(g, m) \in G$ of prime order, say $(g, m)^{p}=1$. Hence $U=\langle g\rangle$ is a subgroup of order $p$ in $F$ and therefore conjugate to $H_{i}$ for some $i$ with $p=p_{i}$. Thus the extension of $M$ by $H_{i}$ splits, and there exists an $m \in M$ with $\left(h_{i}, m\right)^{p_{i}}=1$. Since $M$ is central under the action of $H_{i}$, we can rewrite this as $1=\left(h_{i}, m\right)^{p_{i}}=\left(h_{i}, 1\right)^{p_{i}}(1, m)^{p_{i}}$. In other words, $\left(h_{i}, 1\right)^{p_{i}} \in M^{p_{i}}$, as desired. The converse is obvious.
Let $P(T)$ be the parameterized polycyclic presentation defined by a consistent polycyclic presentation of $F$ and the $F$-module $M$. In computing $Z^{2}(F, M)$, we compute a set of linear conditions on $T$ whose solution space yields $\phi\left(Z^{2}(F, M)\right) \leq M^{l}$. Here we extend this procedure for our purposes using the fact that the condition in Lemma 3.1 can be checked within $P(T)$ as follows.

First, we determine the conjugacy classes of finite subgroups of $F$ using the polycyclic-groups method of [Eick 01a]. If we find a non-cyclic finite subgroup in $F$, then Lemma 3.1 (i) yields that no torsion-free extension exists. If all finite subgroups are cyclic, then we consider the finite subgroups of prime order with representatives $\left\{H_{1}, \ldots, H_{s}\right\}$ in $F$. By Lemma 3.1 ii), we obtain that each extension of $M$ by $F$ contains torsion if one of the subgroups $H_{i}$ is acting nontrivially on $M$. In this case, we can stop the computation, returning that no torsion-free extension exists.

Thus we assume now that all subgroups $H_{i}$ centralize $M$. We apply Lemma 3.1 (iii) to check whether a torsionfree extension of $M$ by $F$ exists. For this purpose, we use the collection algorithm in $P(T)$ to determine a collected word for each element $\left(h_{i}, 1\right)^{p_{i}} \in P(T)$. Since $h_{i}^{p_{i}}=1$ in $F$, we obtain that such a collected word is of the form $w_{i}(T)=T_{1}^{f_{i, 1}} \cdots T_{l}^{f_{i, l}}$ with $f_{i, j} \in \mathbb{Z} F$. As indicated by Lemma 3.1 (iii), we now solve the equations $w_{i}(T) \equiv$ $1 \bmod M^{p_{i}}$ for $1 \leq i \leq s$ over $\phi\left(Z^{2}(F, M)\right) \leq M^{l}$. For each of these equations, we obtain a solution subgroup $S_{i} \leq \phi\left(Z^{2}(F, M)\right) \leq M^{l}$. These yield the desired result as summarized in the following lemma.

Lemma 3.2. Let $M \cong \mathbb{Z}$ be an F-module. Let $\left\{H_{1}, \ldots, H_{s}\right\}$ be a set of conjugacy class representatives of the subgroups of prime order in $F$ and let $S_{1}, \ldots, S_{s}$ be their corresponding subgroups of $\phi\left(Z^{2}(F, M)\right)$ determined as above.

(i) Let $t \in \phi\left(Z^{2}(F, M)\right)$ and let $G_{t}$ be the extension of $M$ by $F$ defined by $P(t)$. Then the group $G_{t}$ is torsion-free if and only if $t \notin \bigcup_{i=1}^{l} S_{i}$.

(ii) Denote o $=\operatorname{lcm}\left\{\left|H_{i}\right| \mid 1 \leq i \leq s\right\}$. Then $\phi\left(Z^{2}(F, M)\right)^{o} \leq S_{i}$ for $1 \leq i \leq s$.

Proof: Part (i) is an immediate consequence of the above discussion and Lemma 3.1. Part (ii) follows directly from the construction of the subgroups $S_{1}, \ldots, S_{s}$.

Thus torsion-free extensions of $M$ by $F$ exist if and only if $\cup_{i=1}^{s} S_{i} / S \neq \phi\left(Z^{2}(F, M)\right) / S$ for $S=\bigcap_{i=1}^{s} S_{i}$. 
Since each $S_{i}$ has finite index in $\phi\left(Z^{2}(F, M)\right)$, their intersection $S$ has finite index as well. Hence it remains to solve a covering problem in a finite group. A method to determine the cardinality of $\cup_{i=1}^{s} S_{i} / S$ can be obtained from the well-known inclusion-exclusion principle based on $|A \cup B|=|A|+|B|-|A \cap B|$ for finite sets $A$ and $B$.

We summarize the results obtained in this section in the following theorem. An extended version of this theorem for $M \cong \mathbb{Z}^{d}$ is also described in [Dekimpe and Eick 01b].

Theorem 3.3. Let $F$ be a polycyclic group and $M$ an $F$-module with $M \cong \mathbb{Z}$. Then there exists an algorithm to check if there exists a torsion-free extension $G$ of $M$ by $F$.

\subsection{Extensions with a Minimal Fitting Centre}

Let $F$ be a polycyclic group with an $F$-module $M$ of the form $M \cong \mathbb{Z}$ such that $\operatorname{Fitt}(F)$ centralizes $M$. Then each extension $G$ of $M$ by $F$ satisfies $M \leq \operatorname{Fitt}(G)$ and $\operatorname{Fitt}(G) / M \cong \operatorname{Fitt}(F)$, since $\operatorname{Fitt}(F)$ centralizes $M$. Thus $M \leq Z(\operatorname{Fitt}(G))$. We want to check if an extension $G$ with $M=Z(\operatorname{Fitt}(G))$ exists. The following lemma yields the basis of our approach to this problem.

Lemma 3.4. Let $G$ be an extension of $M \cong \mathbb{Z}$ by $F$ such that $M$ is central under the action of $\operatorname{Fitt}(F)$. As above, we denote $G=\{(f, m) \mid f \in F, m \in M\}$ and we identify $M$ with $\{(1, m) \mid m \in M\}$. We write $\operatorname{Fitt}(F)=\left\langle a_{1}, \ldots, a_{s}\right\rangle$ and we consider a minimal generating set $b_{1}, \ldots, b_{r}$ of $Z(\operatorname{Fitt}(F))$. We define $c_{i, j}=$ $\left[\left(b_{i}, 1\right),\left(a_{j}, 1\right)\right] \in M$. Then $Z(\operatorname{Fitt}(G))=M$ if and only if the matrix $\left(c_{i, j}\right) \in M^{r \times s} \cong \mathbb{Z}^{r \times s}$ has rank $r$.

Proof: Suppose that $(b, m) \in Z(\operatorname{Fitt}(G))$ with $b=$ $b_{1}^{e_{1}} \cdots b_{r}^{e_{r}}$ and $m \in M$. Then $1=\left[(b, m),\left(a_{j}, 1\right)\right]=$ $c_{1, j}^{e_{1}} \cdots c_{r, j}^{e_{j}}$ and $\left(e_{1}, \ldots, e_{r}\right)$ is in the nullspace of $\left(c_{i, j}\right)$. A nontrivial vector $\left(e_{1}, \ldots, e_{r}\right)$ exists if and only if $r k\left(c_{i, j}\right)<r$.

We use Lemma 3.4 to check if an extension $G$ of $M$ by $F$ with $M=Z(\operatorname{Fitt}(G))$ exists. For this purpose, we first determine generators $a_{1}, \ldots, a_{s}$ for $\operatorname{Fitt}(F)$ and generators $b_{1}, \ldots, b_{r}$ for $Z(\operatorname{Fitt}(F))$ using the polycyclic-groups methods of [Eick 01b]. Then we collect the elements $c_{i, j}$ in a parameterized polycyclic presentation $P(T)$ obtained by extending a consistent polycyclic presentation of $F$.

Each element $c_{i, j}$ has a collected form of the type $c_{i, j}(T)=T_{1}^{f_{i, j, 1}} \cdots T_{l}^{f_{i, j, l}}$ with $f_{i, j, l} \in \mathbb{Z} F$, since $\left[b_{i}, a_{j}\right]=$ 1 in $F$. The action of $F$ on $M$ is given via a homo- morphism $F \rightarrow G L(1, \mathbb{Z}) \cong\{-1,1\}$ and hence we can identify each element $f_{i, j, l} \in \mathbb{Z} F$ with an element in $\mathbb{Z}$. Switching to additive notation, we can identify $c_{i, j}(T)$ with a $\mathbb{Z}$-linear combination of the indeterminates in $T$. We collect these linear combinations in a matrix $F(T)=\left(c_{i, j}(T)\right)$.

We have to check whether there exists a vector $t \in$ $\phi\left(Z^{2}(F, M)\right) \leq M^{l}$ such that $F(t)$ has rank $r$. First we determine a generating set of $\phi\left(Z^{2}(F, M)\right)$ of, say length $m$, and rewrite $F(T)$ such that it becomes a matrix in $m$ indeterminates $\bar{T}_{1}, \ldots, \bar{T}_{m}$ which correspond to the generators of $\phi\left(Z^{2}(F, M)\right)$. Recall that $F(\bar{T})$ is a $(r \times s)$ matrix. If $s<r$, then for all $t$ we obtain that $F(t)$ cannot have rank $r$. Hence we assume in the following that $s \geq r$.

Lemma 3.5. Let $F_{1}(\bar{T}), \ldots, F_{e}(\bar{T})$ be the set of all $r \times r$ submatrices in $F(\bar{T})$. Then there exists a $t \in \phi\left(Z^{2}(F, M)\right)$ with $r k F(t)=r$ if and only if $\sum_{i=1}^{e}\left(\operatorname{det}\left(F_{i}(\bar{T})\right)\right)^{2} \neq 0$.

Proof: $F(t)$ has rank $r$ if and only if one of its $r \times r$ submatrices $F_{i}(t)$ has rank $r$. In turn, $r k F_{i}(t)=r$ if and only if $\operatorname{det}\left(F_{i}(t)\right) \neq 0$. Consider the polynomial $f(\bar{T})=$ $\sum_{i=1}^{e}\left(\operatorname{det}\left(F_{i}(\bar{T})\right)\right)^{2}$ in the indeterminates in $\bar{T}$. If $f(\bar{T}) \neq$ 0 , then there exist values $t \in M^{l}=\mathbb{Z}^{l}$ such that $f(t) \neq 0$. Thus $\left(\operatorname{det}\left(F_{i}(t)\right)\right)^{2} \neq 0$ for at least one $i$. And hence $\operatorname{det}\left(F_{i}(t)\right) \neq 0$ as well.

In the special case that $\operatorname{Fitt}(F)$ is abelian, we can choose the considered generators such that $r=s$ and $b_{i}=a_{i}$. Then $F(T)$ is a $(r \times r)$-matrix satisfying $f_{i, j}(T)=-f_{j, i}(T)$. Thus $F(t)$ has rank $r$ for some $t$ if and only if $\operatorname{det}(F(\bar{T})) \neq 0$. It remains to determine the multivariate polynomial $\operatorname{det}(F(\bar{T}))$ and check if it is identically zero.

In summary, we have proved the following theorem in this section.

Theorem 3.6. Let $F$ be a polycyclic group and $M \cong \mathbb{Z} a$ $\mathbb{Z} F$-module which is centralized by $\operatorname{Fitt}(F)$. Then there exists an algorithm to check whether there exists an extension $G$ of $F$ by $M$ with $Z(\operatorname{Fitt}(G))=M$.

\subsection{Torsion-Free Extensions with a Minimal Fitting Centre}

In this part, we will combine the results of the two previous sections. We show for a given $F$-module $M \cong \mathbb{Z}$ that if there exists a torsion-free extension of $M$ by $F$ and an extension $G$ with $Z(\operatorname{Fitt}(G))=M$, then there also exists an extension $E$ which is both torsion-free and satisfies 
$Z(\operatorname{Fitt}(E))=M$. In the proof of this observation, we use the following lemma.

Lemma 3.7. Let $f\left(T_{1}, \ldots, T_{m}\right)$ be a nonzero polynomial in $m$ variables over $\mathbb{C}$. Further, let $a_{1}, \ldots, a_{m} \in \mathbb{Z}$ and $o_{1}, \ldots, o_{m} \in \mathbb{N}$. Then there exist $b_{1}, \ldots, b_{m} \in \mathbb{Z}$ such that $f\left(b_{1}, \ldots, b_{m}\right) \neq 0$ and $b_{i} \equiv a_{i} \bmod o_{i}$ for all $i \in$ $\{1,2, \ldots, n\}$.

Proof: This lemma is easily proved by induction on the number of variables, $m$.

Theorem 3.8. Let $F$ be a polycyclic group and let $M \cong \mathbb{Z}$ be an $F$-module which is centralized by $\operatorname{Fitt}(F)$. If $G_{1}$ and $G_{2}$ are two extensions of $M$ by $F$ such that $G_{1}$ is torsion-free and $Z\left(\operatorname{Fitt}\left(G_{2}\right)\right)=M$, then there exists an extension $G$ of $M$ by $F$ such that $G$ is torsion-free and $Z(\operatorname{Fitt}(G))=M$.

Proof: Let $\left\{t_{1}, \ldots, t_{m}\right\}$ be a generating set of $\phi\left(Z^{2}(F, M)\right)$. Using additive notation, we obtain that each $t \in \phi\left(Z^{2}(F, M)\right)$ can be written as a linear combination $t=e_{1} t_{1}+\cdots+e_{m} t_{m}$ with $e_{i} \in \mathbb{Z}$. We denote by $G_{t}$ the extension of $M$ by $F$ defined by $P(t)$.

By Lemma 3.5, there exists a polynomial $f\left(\bar{T}_{1}, \ldots, \bar{T}_{m}\right)$ such that $f\left(e_{1}, \ldots, e_{m}\right) \neq 0$ if and only if $Z\left(\operatorname{Fitt}\left(G_{t}\right)\right)=M$ for $t=e_{1} t_{1}+\cdots+e_{m} t_{m}$. We observe that $f$ is not identically zero, since $G_{2}$ is an extension with $Z\left(\operatorname{Fitt}\left(G_{2}\right)\right)=M$. Further, let $o$ denote the least common multiple of the orders of the finite subgroups of $F$ and let $s=a_{1} t_{1}+\cdots+a_{m} t_{m}$ be an element determining the torsion-free extension $G_{s}=G_{1}$.

By Lemma 3.7, there exist elements $b_{1}, \ldots, b_{m} \in \mathbb{Z}$ such that $f\left(b_{1}, \ldots, b_{m}\right) \neq 0$ and $b_{i} \equiv a_{i} \bmod o$. Let $r=b_{1} t_{1}+\cdots b_{m} t_{m} \in \phi\left(Z^{2}(F, M)\right)$. Then $G_{r}$ fulfills $Z\left(\operatorname{Fitt}\left(G_{r}\right)\right)=M$ and, by Lemma $3.2, G_{r}$ is torsion-free.

\section{COMPUTING BETTI NUMBERS}

As a first application of our computational methods, we will show how to compute some of the (co)homology groups and Betti numbers of a compact $K(G, 1)$ manifold, in case $G$ is a torsion-free polycyclic group. If the Hirsch length of $G$ is at most 6 , then we can compute all Betti numbers of the corresponding manifold $K(G, 1)$. For details on Betti numbers we refer the reader to [Brown 82].

Let $G$ be a fixed torsion-free polycyclic-by-finite group and let $M$ be a compact $K(G, 1)$-manifold. Recall that there exists such a $M$ and $\operatorname{dim}(M)=h(G)$. It is well known that the (co)homology of the manifold $M$ coincides with the (co)homology of the group $G$, and therefore the $i$-th Betti number of $M$ is given by

$$
\beta_{i}(M)=\operatorname{rank} H_{i}(G, \mathbb{Z})=\operatorname{rank} H^{i}(G, \mathbb{Z})
$$

where $\mathbb{Z}$ is a trivial $G$-module and rank denotes the torsion-free rank of an abelian group. Moreover, the Euler characteristic of $M$ is the same as the Euler characteristic of the group $G$ and is given by

$$
\chi(M)=\chi(G)=\sum_{i=0}^{h(G)}(-1)^{i} \beta_{i}(M) .
$$

Lemma 4.1. Let $G$ be a nontrivial torsion-free polycyclicby-finite group, then $\chi(G)=0$.

Proof: Let us first prove this result for groups which are poly- $\mathbb{Z}$. If $h(G)=1$, then $G \cong \mathbb{Z}$ and the lemma is obvious. If $h(G)>1$, we can find a normal subgroup $N$ of $G$ with $G / N \cong \mathbb{Z}$. It is known (see [Brown 82]) that $\chi(G)=\chi(N) \chi(\mathbb{Z})=0$. If $G$ is a more general torsion-free polycyclic-by-finite group, we can find a normal subgroup $N$ which is of finite index in $G$ and which is poly- $\mathbb{Z}$. Using the fact that $\chi(G)=\chi(N) /[G: N]$, we find that $\chi(G)=0$.

For an $n$-dimensional compact $K(G, 1)$ manifold $M$, there is a nice connection between the $i$-th cohomology groups and the $(n-i)$-th homology groups. Let $\tilde{M}$ be the universal covering manifold of $M$. Then $\tilde{M}$ is orientable and we denote the orientation module of $\tilde{M}$ by $D$. The module $D$ is isomorphic to $\mathbb{Z}$ and the action of an element $g \in G$ on $\tilde{M}$ induces an action on $D$ as follows: $g$ acts as +1 (resp. -1) on $D$ iff the action of $g$ on $\tilde{M}$ is orientation preserving (respectively reversing). So, $G$ acts trivially on $\tilde{M}$ if and only if $M$ is orientable. We now have the following lemma ([Brown 82], [Dekimpe 96]):

Lemma 4.2. Let $G$ be the fundamental group of a compact $n$-dimensional $K(G, 1)$-manifold with associated orientation module $D$ and let $\mathbb{Z}$ be the trivial $G$-module, then

$$
\forall i \in\{0,1,2, \ldots, n\}: H^{i}(G, D) \cong H_{n-i}(G, \mathbb{Z}) .
$$

To make use of this lemma, we need to be able to compute the action of $G$ on $D$. For this purpose, we determine a series

$G_{*}: G=G_{1} \triangleright G_{2} \triangleright \ldots \triangleright G_{c} \triangleright G_{c+1}=1 \quad$ (for some $c$ ) 
of normal subgroups of $G$ whose factors are either free abelian or finite. Such a sequence of normal subgroups exists (but is far from unique) in each polycyclic-by-finite group. Let us refer to such a normal series as a ffa-series (finite or free abelian quotients). As examples of such ffa-series, we mention the torsion-free filtrations used in [Dekimpe 96] and [Dekimpe and Igodt 97] and the efaseries (elementary or free abelian quotients) which can be computed effectively using the methods in [Eick 01a] or [Eick $01 \mathrm{~b}]$.

Let $J \subseteq\{1, \ldots, c\}$ be the set of those indices with $G_{i} / G_{i+1}$ free abelian. Each element $g \in G$ induces an automorphism on each free abelian quotient $G_{i} / G_{i+1}$ by conjugation. Choosing free generators for $G_{i} / G_{i+1}$, we can describe this automorphism as a matrix $A_{i}(g) \in$ $\operatorname{Aut}\left(G_{i} / G_{i+1}\right)$. Thus, for each $g \in G$, we can define

$$
\operatorname{or}(g)=\prod_{i \in J} \operatorname{det}\left(A_{i}(g)\right) \in\{1,-1\}
$$

If $J=\emptyset$ and thus $G$ is finite, then we let $\operatorname{or}(g)=1$ for all $g \in G$. This number or $(g)$ is independent of the choice of generators for the free abelian quotients $G_{i} / G_{i+1}$. Further, $\operatorname{or}(g)$ is also independent on the choice of the ffaseries $G_{*}$ of $G$. This can be observed easily using the fact that or $(g)$ defines the action of $G$ on the orientation module $D$ and the results of the following theorem.

Theorem 4.3. Let $G$ be any polycyclic-by-finite group (not necessarily torsion-free) equipped with a ffa-series $G_{*}$. Then $G$ admits a properly discontinuous action on $\mathbb{R}^{h(G)}$ such that the action of any element $g \in G$ is orientation preserving if and only if or $(g)=1$.

Proof: We proceed by induction on the length $c$ of the ffa-series $G_{*}$ of $G$. If this length $c=0$, the group $G$ is finite and so $h(G)=0, \mathbb{R}^{h(G)}$ is a point and the trivial action of $G$ on this point satisfies the statement of the theorem.

Assume now that $c>1$ and that the theorem is valid for polycyclic-by-finite groups having a ffa-series of smaller length. Let $G_{*}$ (as in $(4-1)$ ) be a ffa-series of length $c$ for $G$. Then we denote $\bar{G}=G / G_{c}$ and note that the induced series

$$
\begin{aligned}
\bar{G}_{*}: \bar{G}_{1} & =\bar{G} \triangleright \bar{G}_{2}=G_{2} / G_{c} \triangleright \cdots \triangleright \bar{G}_{c-1} \\
& =G_{c-1} / G_{c} \triangleright \bar{G}_{c}=G_{c} / G_{c}=1
\end{aligned}
$$

is a ffa-series of $\bar{G}$ of length $c-1$. We also denote the natural projection of an element $g \in G$ to $\bar{G}$ by $\bar{g}$. We now distinguish two cases.
Case 1: $G_{c}$ is finite. In this case, $\operatorname{or}(g)=\operatorname{or}(\bar{g})$ for all $g \in G$. (where, of course, or $(g)$ is computed via $G_{*}$ and $\operatorname{or}(\bar{g})$ is obtained form $\left.\bar{G}_{*}\right)$. Moreover, by the induction hypothesis, we know that $\bar{G}$ admits a properly discontinuous action on $\mathbb{R}^{h(\bar{G})}$ such that the action of $\bar{g}$ is orientation-preserving if and only if $\operatorname{or}(\bar{g})=1$. As $h(G)=h(\bar{G})$, we can use this action to let $G$ act on $\mathbb{R}^{h(G)}$ and the conditions of the theorem will be satisfied.

Case 2: $G_{c}$ is free abelian. In this case, $G_{c} \cong \mathbb{Z}^{k_{c}}$ and one can easily observe that $\operatorname{or}(g)=\operatorname{det}\left(A_{c}(g)\right) \cdot \operatorname{or}(\bar{g})$. By the induction hypothesis, we know that there exists a properly discontinuous action of $\bar{G}$ on $\mathbb{R}^{h(\bar{G})}$ such that for any $\bar{g} \in \bar{G}$, or $(\bar{g})= \pm 1$ depending upon the fact whether or not the action of $\bar{g}$ is orientation-preserving or not. From the work of P. Conner and F. Raymond on Seifert Fibre Spaces (see [Conner and Raymond 71], [Conner and Raymond 77], but be careful because in this work left actions are used), we know that there exists a properly discontinuous action of $G$ on $\mathbb{R}^{k_{c}} \times \mathbb{R}^{h(\bar{G})}=\mathbb{R}^{h(G)}$, such that $g$ acts in the following way:

$$
\forall x \in \mathbb{R}^{k_{c}}, \forall y \in \mathbb{R}^{h(\bar{G})}:(x, y)^{g}=\left(x A_{c}(g)+h_{g}(y), y^{\bar{g}}\right),
$$

for some map $h_{g}: \mathbb{R}^{h(\bar{G})} \rightarrow \mathbb{R}^{k_{c}}$. It is now obvious that the action of $g$ is orientation-preserving if and only if $\operatorname{det}\left(A_{c}(g)\right) \cdot \operatorname{or}(\bar{g})=1=\operatorname{or}(g)$.

Remark 4.4. If $G$ is a torsion-free polycyclic group, the action constructed in Theorem 4.3 will be fixed-point free and the quotient space $\mathbb{R}^{h(G)} / G$ will be a compact $K(G, 1)$-manifold, with universal cover $\mathbb{R}^{h(G)}$. The action of an element $g \in G$ on the orientation module $D$ is therefore given by $\operatorname{or}(g)$.

Definition 4.5. For any polycyclic-by-finite group $G$ (not necessarily torsion-free), we will say that $G$ is orientable iff $\forall g \in G:$ or $(g)=+1$

Theorem 4.6. Let $G$ be a torsion-free polycyclic group of Hirsch length $h(G)=n$, then we can compute $\beta_{i}(G)$ for $i \in\{0,1, n-2, n-1, n\}$ using the following descriptions for these Betti numbers.

\begin{tabular}{l||l|l|l}
$i$ & 0 & 1 & 2 \\
\hline$\beta_{i}(G)$ & 1 & $\operatorname{rank} G / G^{\prime}$ & $\operatorname{rank} H^{2}(G, \mathbb{Z})$ \\
\hline \hline$i$ & $n-2$ & $n-1$ & $n$ \\
\hline$\beta_{i}(G)$ & $\operatorname{rank} H^{2}(G, D)$ & $\operatorname{rank} H^{1}(G, D)$ & $\operatorname{rank} H^{0}(G, D)$
\end{tabular}

Thus, if $n \leq 5$, then this yields a method to determine all Betti numbers of $G$. Additionally, if $n=6$, then we also can compute all Betti numbers of $G$ using the Euler characteristic. 
Proof: For each $G$, we have $H_{0}(G) \cong \mathbb{Z}$ and $H_{1}(G)=$ $G /[G, G]$ yielding $\beta_{i}(G)$ for $i=0,1$. The second Betti number $\beta_{2}$ is by definition the rank of $H^{2}(G, \mathbb{Z})$. Then using $H_{n-i}(G)=H^{i}(G, D)$ yields $\beta_{i}(G)$ for $i=n-2, n-$ $1, n$. Finally, for the case $n=6$, we can compute $\beta_{3}$ using the fact that the Euler characteristic of $G$ is 0 .

Note that $\beta_{1}$ can also be computed as the rank of $H^{1}(G, \mathbb{Z})$.

An algorithm to determine the Betti numbers $\beta_{0}, \beta_{1}, \beta_{n-1}$ and $\beta_{n}$ for virtually nilpotent groups has also been described in [Dekimpe 96].

Example 4.7. This method can be used to determine the Betti numbers for all torsion-free polycyclic 4dimensional crystallographic groups. On average, such a computation takes about 0.1 to $0.5 \mathrm{sec}$. For example, it takes $0.1 \mathrm{sec}$ to determine the Betti numbers

$$
\beta(G)=(1,0,1,2,0)
$$

for the 4-dimensional crystallographic group $G$ of type 04/03/01/06 ([Brown et al. 78]) . This group is torsionfree and its point group is the elementary abelian group of order 4. Its orientation module is nontrivial.

\section{ALMOST BIEBERBACH GROUPS}

Another area where we can apply our computational knowledge is in the study of almost crystallographic groups. Let us briefly recall the setting in which these groups arise. The reader can find details and more information in [Dekimpe 96] and its references. Although one mostly uses left actions in this setting, we continue to use right actions, in order to remain consistent with the rest of the paper. Let $L$ be a connected and simply connected nilpotent Lie group and let $\operatorname{Aff}(L)=\operatorname{Aut}(L) \ltimes L$ be the affine group of $L$. There is a natural right action of $\operatorname{Aff}(L)$ on $L$ :

$$
\forall l, l^{\prime} \in L, \forall \alpha \in \operatorname{Aut}(L): l^{\left(\alpha, l^{\prime}\right)}=l^{\alpha} l^{\prime} .
$$

Definition 5.1. Let $C$ be a compact subgroup of $\operatorname{Aut}(L)$. Then a discrete and cocompact subgroup $G$ of $C \ltimes L$ is called an almost crystallographic group. Moreover, if $G$ is torsion-free, the group $G$ is said to be almost Bieberbach.

Note that for $L=\mathbb{R}^{n}$ and $C=O(n)$ (the orthogonal group), the almost crystallographic (respectively Bieberbach) groups are exactly the classical crystallographic (respectively Bieberbach) groups.
If $G$ is an almost Bieberbach group, the quotient space $L / G$ is a manifold which is called an infra-nilmanifold. For any almost crystallographic group $G, N=G \cap L$ is a uniform lattice of $L$ and $F=G / N$ is a finite group, the holonomy group of $G$. It follows that $N$ is a finitely generated torsion-free nilpotent group. Moreover, $N$ is a maximal nilpotent subgroup of $G$ and hence $N=\operatorname{Fitt}(G)$.

Definition 5.2. Let $N$ be a finitely generated, torsionfree nilpotent group. A group extension $1 \rightarrow N \rightarrow G \rightarrow$ $F \rightarrow 1$ is said to be essential if $N$ is maximal nilpotent in $G$ and $F$ is finite.

Every almost crystallographic group fits into an essential extension. But also the converse is true: Every group $G$ arising in an essential extension can be realized as an almost crystallographic group. The Lie group $L$ needed for this $G$ will be the Mal'cev completion of $N=\operatorname{Fitt}(G)$.

In [Dekimpe 96], all almost Bieberbach groups of Hirsch length at most 4 have been classified. A key observation for this classification is due to K.B. Lee and it uses the notion of isolators defined as follows:

Definition 5.3. Let $G$ be a group and $H \leq G$. The isolator of $H$ in $G$ is defined by

$$
\sqrt[G]{H}=\left\{g \in G \mid g^{k} \in H \text { for some integer } k>0\right\} .
$$

If $G$ is any group, then $\sqrt[G]{\gamma_{c}(G)}$ is a characteristic subgroup of $G$ and $G / \sqrt[G]{\gamma_{c}(G)}$ is nilpotent of class at most $c-1$. We can now formulate Lee's observation.

Lemma 5.4. ([Lee 88] and [Dekimpe 96, Lemma 2.4.2]) Let $N$ be a finitely generated, torsion-free c-step nilpotent group with $c>1$. Define $Z=\sqrt[N]{\gamma_{c}(N)}$. Then for any finite group $F$,

$$
\begin{aligned}
1 \rightarrow N \rightarrow G \rightarrow & F \rightarrow 1 \text { is essential } \\
& \mathbb{1} \\
1 \rightarrow N / Z \rightarrow G / Z & \rightarrow F \rightarrow 1 \text { is essential. }
\end{aligned}
$$

This lemma yields the commutative diagram shown in Figure 1. The commutative diagram shows that any almost crystallographic group $G$ with Fitting subgroup $N$ arises as an extension $1 \rightarrow Z \rightarrow G \rightarrow G / Z \rightarrow 1$ where $G / Z$ is an almost crystallographic group having $N / Z$ as Fitting subgroup. Moreover, this extension induces an action of $G / Z$ on $Z$ by conjugation in $G$ in such a way that $N / Z$ acts trivially on $Z$. Therefore, the action of $G / Z$ on $Z$ factors through the finite holonomy group $F$. 


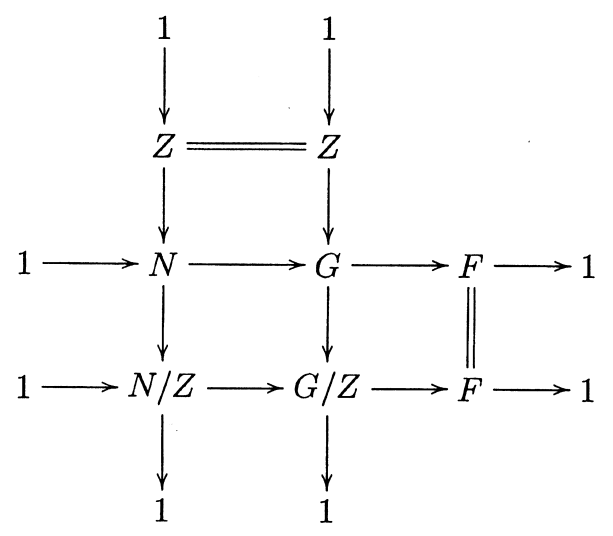

FIGURE 1. Reduction diagram.

\subsection{Classifying 4-Dimensional Almost Bieberbach Groups, Revisited}

A classification of all almost Bieberbach groups of Hirsch length at most 4 was obtained in [Dekimpe 96]. By a "classification," we mean a list containing all possible isomorphism types exactly once. In this section, we explain how most of the work of this classification can now be redone in an automatic way. Moreover, we found that there is one family of almost Bieberbach groups which is missing in [Dekimpe 96].

An almost Bieberbach group $G$ of Hirsch length 4 fits into an essential extension $1 \rightarrow N \rightarrow G \rightarrow F \rightarrow 1$, where $N$ is a nilpotent group of Hirsch length 4. If $N$ is abelian, then $G$ is a 4-dimensional crystallographic group. These crystallographic groups have been classified in [Brown et al. 78]. Thus we can assume here that $N$ is nilpotent, but nonabelian, and hence $N$ can be of class 2 or 3 . The case, $N$ is of class 3 , is a small case that can be dealt with readily. We refer to [Dekimpe 96] for details on this case.

In the following, we consider the case that $N$ is of class 2, and we show that the almost Bieberbach groups $G$ having such a group $N$ as Fitting subgroup can be determined using the algorithmic methods of the first part of this paper. We summarize the properties of $N$ and $G$ in this case in the following:

Lemma 5.5. Let $G$ be an almost Bieberbach group with $N=\operatorname{Fitt}(G)$ of class 2 and Hirsch length 4. Then

- $Z=\sqrt[N]{[N, N]} \cong \mathbb{Z}$

- $Q=G / Z$ is a 3-dimensional crystallographic group whose finite subgroups are cyclic.
- Each element of finite order in $Q$ acts trivially on $Z$ and $\operatorname{Fitt}(Q)$ centralizes $Z$.

Proof: The group $N$ is a torsion-free nilpotent group of Hirsch length 4 and class 2 . Thus $[N, N] \cong \mathbb{Z}$ and also $\sqrt[N]{[N, N]} \cong \mathbb{Z}$. By the definition of isolators, this yields $N / Z \cong \mathbb{Z}^{3}$. By Lemma $5.4, Q=G / Z$ is a crystallographic group of dimension 3. Further, using Lemma 3.1, we observe that all finite subgroups of $Q$ must be cyclic and the elements of finite order in $Q$ act trivially on $Z$. Clearly, also $\operatorname{Fitt}(Q)$ centralizes $Z$.

These properties can be used to obtain an algorithm to determine all 4-dimensional almost Bieberbach groups with such a group $N$ as Fitting subgroup as follows: We start with a list of isomorphism-type representatives for the 3-dimensional crystallographic groups $Q$. Note that these groups are all polycyclic, and thus we can apply the methods of Section 3. to determine their cohomology groups and check for torsion-free extensions with a module $Z \cong \mathbb{Z}$. By Lemma 5.4, each such extension defines an almost Bieberbach group $G$ and the Fitting subgroup of $G$ has Hirsch length 4. It remains to reduce to those extensions whose Fitting subgroup has class 2; that is, whose Fitting subgroup is nonabelian. A test for this purpose is obtained from the following lemma.

Lemma 5.6. Let $Q$ be a group containing a free abelian group $T \cong \mathbb{Z}^{n}$ of finite index. Let $\varphi: Q \rightarrow \operatorname{Aut}\left(\mathbb{Z}^{k}\right)$ be an action of $Q$ on $\mathbb{Z}^{k}$ with $\varphi(T)=1$. Assume that $\alpha \in H^{2}\left(Q, \mathbb{Z}^{k}\right)$ determines an extension $1 \rightarrow \mathbb{Z}^{k} \rightarrow G \stackrel{p}{\rightarrow}$ $Q \rightarrow 1$. Then the full preimage $p^{-1}(T)$ is abelian if and only if $\alpha$ is of finite order in $H^{2}\left(Q, \mathbb{Z}^{k}\right)$.

Proof: The induced extension

$$
1 \rightarrow \mathbb{Z}^{k} \rightarrow p^{-1}(T) \rightarrow T \rightarrow 1
$$

corresponds to $\operatorname{res}(\alpha) \in H^{2}\left(T, \mathbb{Z}^{k}\right) \cong \mathbb{Z}^{k \frac{n(n-1)}{2}}$, where res denotes the well-known restriction map res: $H_{\varphi}^{2}\left(Q, \mathbb{Z}^{k}\right) \rightarrow$ $H^{2}\left(T, \mathbb{Z}^{k}\right)$. It is easy to see that a (central) extension of $T$ by $\mathbb{Z}^{k}$ is abelian if and only if it corresponds to the zero element of $H^{2}\left(T, \mathbb{Z}^{k}\right)$. Thus the extension $(5-1)$ is abelian if and only if $\operatorname{res}(\alpha)=0$.

As $T$ is of finite index in $Q$, we can also consider the corestriction map cor: $H^{2}\left(T, \mathbb{Z}^{k}\right) \rightarrow H^{2}\left(Q, \mathbb{Z}^{k}\right)$ and we know that the composition cor $\circ$ res is just multiplication by the index $[Q: T]$.

Hence, if $p^{-1}(T)$ is abelian, (cor $\circ$ res $)(\alpha)=\operatorname{cor}(0)=0$ showing that the order of $\alpha$ is finite. On the other hand, if the order of $\alpha$ is finite, then necessarily $\operatorname{res}(\alpha)=0$, showing that $p^{-1}(T)$ is abelian. 


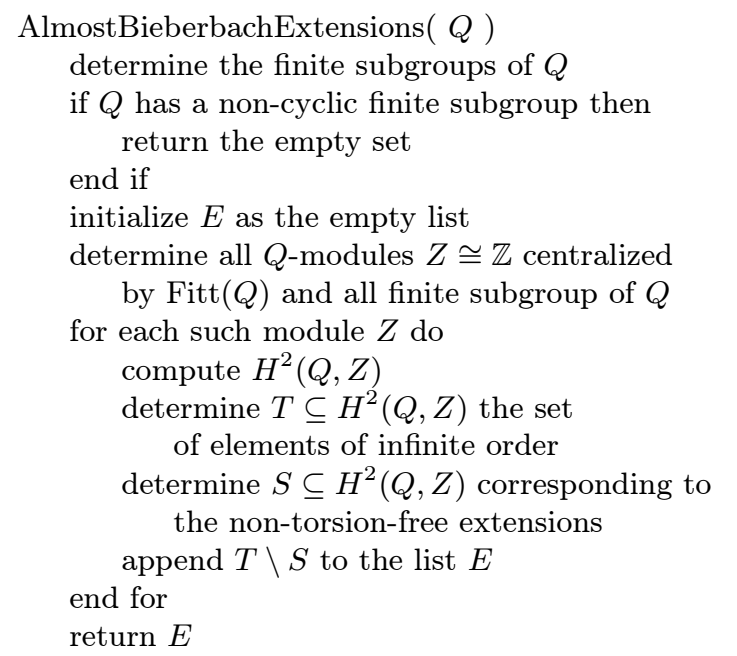

FIGURE 2. Determining almost Bieberbach extensions.

We determine the almost Bieberbach groups $G$ whose Fitting subgroup $N$ is of class 2 and Hirsch length 4 by applying the methods in Figure 2 to the 219 representatives for the 3-dimensional crystallographic groups $Q$.

Within this method, we determine the finite subgroups of the polycyclic group $Q$ as described in [Eick 01a]. Further, the desired modules $Z$ can be computed as discussed in the beginning of Section 3. Since $H^{2}(Q, Z)$ is a finitely generated abelian group, it contains a torsion subgroup $A$. Each element of infinite order in $H^{2}(Q, Z)$ is contained in a complement to $A$ in $H^{2}(Q, Z)$. Thus the set $T$ of all elements of infinite order can be determined as the union of the (finitely many) complements to $A$ in $H^{2}(Q, Z)$. Further, the set $S$ describing the non-torsionfree extensions is a union of subgroups of $H^{2}(Q, Z)$ as observed in Section 3.1. Thus $T$ and $S$ can be determined explicitly and $T \backslash S$ corresponds to the set of the desired almost Bieberbach extensions.

Remark 5.7. In applying the algorithm "AlmostBieberbachExtensions" to classify extensions, it remains to reduce the obtained list of extensions to isomorphism type representatives. For this purpose, we use case-by-case arguments.

In applying this algorithm, we consider the 219 representatives of the 3-dimensional crystallographic groups. First, we observe that 84 of them have only cyclic finite subgroups. For these 84 groups, we obtain a list of 74 modules $Z$ which lead to almost Bieberbach extensions of the desired type. A table containing more detailed information on these 74 modules is given in Appendix A.

\subsection{Infra-Nilmanifolds Modeled on Heisenberg Lie Groups}

Recently, there has been interest in infra-nilmanifolds modeled on Heisenberg Lie groups ([Dekimpe et al. 95], [Lee 00], [Lee and Szczepański 00]). These are built using almost Bieberbach groups living inside the affine group $\operatorname{Aff}(L)$ of a Heisenberg Lie group.

\section{Definition 5.8.}

a) A connected and simply connected Lie group $L$ is said to be a Heisenberg Lie group if $L$ is 2-step nilpotent and $Z(L)=[L, L]$ is 1-dimensional.

b) A Lie algebra $\mathfrak{l}$ over a field $F$ is said to be a Heisenberg Lie algebra if and only if $\mathfrak{l}$ is 2 -step nilpotent and $Z(\mathfrak{l})=[\mathfrak{l}, \mathfrak{l}]$ is 1-dimensional.

Of course, a connected and simply connected Lie group is Heisenberg if and only if the corresponding Lie algebra is Heisenberg.

In this section, we will first examine these Heisenberg Lie groups in more detail and then apply this knowledge, together with our computational method, to show that the results of [Lee and Szczepański 00] and [Lee 00] can be checked and extended using our algorithmic methods.

5.2.1 Automorphisms of Heisenberg Lie algebras. Proposition 5.9. Let $\mathfrak{l}$ be a Heisenberg Lie algebra over a Field $F$ and let $\varphi \in \operatorname{Aut}(\mathfrak{l})$ be an automorphism which is diagonalizable over $F$. Then there exists a vector space basis $X_{1}, X_{2}, \ldots, X_{n}, Y_{1}, Y_{2}, \ldots, Y_{n}, Z$ of eigenvectors of $\varphi$ with

$$
\begin{gathered}
\forall i:\left[X_{i}, Y_{i}\right]=Z, \forall i, j \text { with } i \neq j:\left[X_{i}, Y_{j}\right]=0, \\
\forall i, j:\left[X_{i}, X_{j}\right]=\left[Y_{i}, Y_{j}\right]=0, \forall i:\left[X_{i}, Z\right]=\left[Y_{i}, Z\right]=0
\end{gathered}
$$

Proof: Let $Z$ be any nonzero vector of $Z(\mathfrak{l})$. As $Z(\mathfrak{l})$ is invariant under $\varphi$ and is also 1-dimensional, we can conclude that $Z$ is an eigenvector of $\varphi$.

Now, let $X_{1}$ be any eigenvector which is linearly independent from $Z$. There must exist another eigenvector $Y_{1}$ of $\varphi$ such that $\left[X_{1}, Y_{1}\right] \neq 0$; otherwise $X_{1}$ would be in the center of $\mathfrak{l}$, which is impossible (remember that $\varphi$ is supposed to be diagonalizable, i.e., has a basis of eigenvectors). By eventually rescaling $Y_{1}$, we may assume that $\left[X_{1}, Y_{1}\right]=Z$.

Now, we construct the space $\mathfrak{l}^{\prime}=\left\{V \in \mathfrak{l} \mid\left[V, X_{1}\right]=\right.$ $\left.\left[V, Y_{1}\right]=0\right\}$. As $\mathfrak{l}^{\prime}$ contains $Z(\mathfrak{l})=[\mathfrak{l}, \mathfrak{l}], \mathfrak{l}^{\prime}$ is a sub Lie algebra of $\mathfrak{l}$. 
Moreover, $\mathfrak{l}^{\prime}=\mathfrak{l}_{1} \cap \mathfrak{l}_{2}$, where

$\mathfrak{l}_{1}=\left\{V \in \mathfrak{l} \mid\left[V, X_{1}\right]=0\right\}$ and $\mathfrak{l}_{2}=\left\{V \in \mathfrak{l} \mid\left[V, Y_{1}\right]=0\right\}$.

Note that $\mathfrak{l}_{1}$ is, in fact, the kernel of the linear map $\lambda$ : $\mathfrak{l} \rightarrow Z(\mathfrak{l}): V \mapsto\left[V, X_{1}\right]$. The image of $\lambda$ is $Z(\mathfrak{l})$, and therefore, the kernel of $\lambda, \mathfrak{l}_{1}$ is of codimension 1 in $\mathfrak{l}$. Analogously, $\mathfrak{l}_{2}$ is of codimension 1 in $\mathfrak{l}$. It follows that $\mathfrak{l}^{\prime}$ is of codimension 2 in $\mathfrak{l}$. In other words, as vector spaces, we have $\mathfrak{l}=\left\langle X_{1}, Y_{1}\right\rangle \oplus \mathfrak{l}^{\prime}$ where $\left\langle X_{1}, Y_{1}\right\rangle$ is the space spanned by $X_{1}$ and $Y_{1}$. Moreover, $\mathfrak{l}^{\prime}$ is again a Heisenberg Lie algebra (note that the center of $\mathfrak{l}^{\prime}$ must coincide which the center of $\mathfrak{l}$ ) which is invariant under $\varphi$. The proof now finishes by an induction argument on the dimension.

Remark 5.10. For the identity automorphism, this proposition is always applicable and, in fact, it shows that our definition of Heisenberg Lie algebras corresponds to the more standard definition used in [Goze and Khkimdjanov 96].

As an immediate consequence of this proposition, we get the following well-known result. (This is, in most cases, assumed in the definition of a Heisenberg Lie algebra.)

Corollary 5.11. Any Heisenberg Lie algebra (or Lie group) is odd-dimensional.

Proposition 5.12. Let $\mathfrak{l}$ be a Heisenberg Lie algebra over $\mathbb{R}$ of dimension $2 k+1$ and let $\varphi \in \operatorname{Aut}(\mathfrak{l})$. Then

- If $k$ is odd, $\varphi$ is an orientation-preserving automorphism of the vector space $\mathfrak{l}$.

- If $k$ is even, $\varphi$ induces an orientation-preserving automorphism on $\mathfrak{l} /[\mathfrak{l}, \mathfrak{l}]$.

Proof: First of all, note that $Z(\mathfrak{l})$ consists of eigenvectors of $\varphi$ with respect to some real eigenvalue $\lambda$.

Next, we consider the complexification $\mathfrak{l}_{\mathbb{C}}=\mathfrak{l} \otimes \mathbb{C}$ of $\mathfrak{l}$. The automorphism $\varphi$ induces, in a unique way, an automorphism $\varphi_{\mathbb{C}}$ of the complex Lie algebra $\mathfrak{l}_{\mathbb{C}}$. Let us denote the induced automorphism on $\mathfrak{l}_{\mathbb{C}} /\left[\mathfrak{l}_{\mathbb{C}}, \mathfrak{l}_{\mathbb{C}}\right]$ by $\bar{\varphi}_{\mathbb{C}}$. We need to show that $\operatorname{det}\left(\varphi_{\mathbb{C}}\right)$ (respectively $\left.\operatorname{det}\left(\bar{\varphi}_{\mathbb{C}}\right)\right)$ is a positive real number when $k$ is odd (respectively even). Using the multiplicative Jordan decomposition of $\varphi_{\mathbb{C}}$, we can write $\varphi_{\mathbb{C}}$ as a product $\varphi_{\mathbb{C}}=$ $\varphi_{\mathbb{C}}^{u} \varphi_{\mathbb{C}}^{s}$ where $\varphi_{\mathbb{C}}^{u}$ is a unipotent automorphism, $\varphi_{\mathbb{C}}^{s}$ is a semi-simple automorphism and $\varphi_{\mathbb{C}}^{u}$ and $\varphi_{\mathbb{C}}^{s}$ commute with each other. It follows that it is enough to prove the proposition for $\varphi_{\mathbb{C}}$ semi-simple, i.e., diagonalizable. In this case, we apply Proposition 5.9 to find a basis $X_{1}, X_{2}, \ldots, X_{k}, Y_{1}, Y_{2}, \ldots, Y_{k}, Z$ of $\mathfrak{l}_{\mathbb{C}}$ consisting of eigenvectors of $\varphi_{\mathbb{C}}$ with

$$
\forall i:\left[X_{i}, Y_{i}\right]=Z, \forall i, j \text { with } i \neq j:\left[X_{i}, Y_{j}\right]=0,
$$

$\forall i, j:\left[X_{i}, X_{j}\right]=\left[Y_{i}, Y_{j}\right]=0, \quad \forall i:\left[X_{i}, Z\right]=\left[Y_{i}, Z\right]=0$.

Note that $\varphi_{\mathbb{C}}(Z)=\lambda Z$ (where $\lambda$ is a real number). Now, assume that $\varphi_{\mathbb{C}}\left(X_{i}\right)=\alpha_{i} X_{i}$ and $\varphi_{\mathbb{C}}\left(Y_{i}\right)=\beta_{i} Y_{i}$ for some complex numbers $\alpha_{i}$ and $\beta_{i}$. Using the fact that $Z=$ $\left[X_{i}, Y_{i}\right]$, we find that

$$
\begin{aligned}
\lambda Z & =\varphi_{\mathbb{C}}(Z)=\varphi_{\mathbb{C}}\left[X_{i}, Y_{i}\right] \\
& =\left[\alpha_{i} X_{i}, \beta_{i} Y_{i}\right]=\alpha_{i} \beta_{i} Z \Rightarrow \alpha_{i} \beta_{i}=\lambda .
\end{aligned}
$$

It follows that

$$
\operatorname{det}\left(\varphi_{\mathbb{C}}\right)=\lambda\left(\alpha_{1} \beta_{1}\right)\left(\alpha_{2} \beta_{2}\right) \ldots\left(\alpha_{k} \beta_{k}\right)=\lambda^{k+1}
$$

and

$$
\operatorname{det}\left(\bar{\varphi}_{\mathbb{C}}\right)=\left(\alpha_{1} \beta_{1}\right)\left(\alpha_{2} \beta_{2}\right) \ldots\left(\alpha_{k} \beta_{k}\right)=\lambda^{k}
$$

proving the proposition.

We apply the results obtained so far to infranilmanifolds and obtain the following description of their underlying fundamental groups.

Lemma 5.13. The group $G$ is the fundamental group of an infra-nilmanifold of dimension $2 k+1$ modeled on a Heisenberg Lie group if and only if

- $Z=Z(\operatorname{Fitt}(G)) \cong \mathbb{Z}$, and

- $Q=G / Z$ is a $2 k$-dimensional crystallographic group, and

- $G$ is torsion-free.

Proof: An essential extension of the form $1 \rightarrow N \rightarrow G \rightarrow$ $F \rightarrow 1$ determines an almost Bieberbach group corresponding to an infra-nilmanifold modeled on a Heisenberg Lie group if and only if $G$ is torsion-free and $N=$ $\operatorname{Fitt}(G)$ is a uniform lattice of a Heisenberg Lie group. By [Segal 83, Proposition 5, page 265], one can see that a group $N$ is a uniform lattice of a Heisenberg Lie group if and only if $N$ is a finitely generated, torsion-free 2step nilpotent group with $Z(N) \cong \mathbb{Z}$. Thus also $Z=$ $\sqrt[N]{[N, N]}=Z(N) \cong \mathbb{Z}$. It follows that any such $G$ fits in a short exact sequence $1 \rightarrow \mathbb{Z} \rightarrow G \rightarrow G / \mathbb{Z} \rightarrow 1$ where $G / \mathbb{Z}$ is an even-dimensional crystallographic group.

Conversely, any extension $1 \rightarrow Z \rightarrow G \rightarrow G / Z \rightarrow 1$ of a free abelian group $Z$ of finite rank by a crystallographic group $G / Z$, satisfying $Z \subseteq Z(\operatorname{Fitt}(G))$, is an 
almost crystallographic group, $\operatorname{since} \operatorname{Fitt}(G)$ will be maximal nilpotent in $G$, as $\operatorname{Fitt}(G / Z)$ is maximal nilpotent in $G / Z$, since $G / Z$ is crystallographic. Further, $G / \operatorname{Fitt}(G) \cong(G / Z) / \operatorname{Fitt}(G / Z)$ is the holonomy group of $G / Z$ which is finite.

As mentioned before, the corresponding nilpotent Lie group of an almost crystallographic group $G$, is the Mal'cev completion of $\operatorname{Fitt}(G)$. By the assumption that $Z=Z(\operatorname{Fitt}(G))=\mathbb{Z}$ and $\operatorname{Fitt}(G) / Z \cong \operatorname{Fitt}(G / Z)$ is abelian $(G / Z$ is crystallographic), we see that the

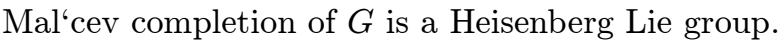

We are now ready to formulate the following result which generalizes both [Dekimpe et al. 95, Corollary 4.1] and [Lee and Szczepański 00, Lemma 5].

Theorem 5.14. Let $M$ be an infra-nilmanifold modeled on a Heisenberg Lie group of dimension $2 k+1$ and let $G$ be the corresponding almost Bieberbach group. Then

- If $k$ is odd then $M$ and $G$ are orientable.

- If $k$ is even, then the underlying crystallographic group of $G$ is orientable.

Proof: This is an immediate consequence of Proposition 5.12 .

Note that the properties of $G$ as outlined above can be checked algorithmically for each crystallographic group $Q$ using the methods of Section 3.

We can therefore very easily now prove (again) and generalize the main result of [Lee and Szczepański 00]:

Theorem 5.15. An infra-nilmanifold modeled on a 5dimensional Heisenberg Lie group has a polycyclic fundamental group whose holonomy group is of order $\leq 24$. More precisely, Table 1 lists the holonomy groups.

Proof: Using GAP we consider each of the 4,783 representatives of the 4-dimensional crystallographic groups in turn and check if they arise as the underlying crystallographic group of the fundamental group of an infranilmanifold modeled on the 5-dimensional Heisenberg Lie group. First we observe that 723 of these crystallographic groups are oriented. We reduce our computations to these groups by Theorem 5.14. In this list of 723 groups, only 6 of them are nonsolvable. In the notation of [Brown et al. 78] they are the groups with types

$$
\begin{aligned}
& 31 / 3 / 1 / 1,31 / 3 / 2 / 1,31 / 6 / 1 / 1,31 / 6 / 2 / 1 \text {, } \\
& 31 / 3 / 1 / 2, \quad 31 / 3 / 2 / 2 \text {. }
\end{aligned}
$$

\begin{tabular}{|c|c|c|}
\hline Order & Holonomy group & Number of occurrences \\
\hline 1 & 1 & 1 \\
2 & $C_{2}$ & 6 \\
3 & $C_{3}$ & 5 \\
4 & $C_{4}$ & 10 \\
4 & $V_{4}$ & 30 \\
5 & $C_{5}$ & 1 \\
6 & $S_{3}$ & 7 \\
6 & $C_{6}$ & 10 \\
8 & $C_{8}$ & 1 \\
8 & $C_{4} \times C_{2}$ & 6 \\
8 & $D_{8}$ & 20 \\
8 & $Q_{8}$ & 1 \\
8 & $C_{2}^{3}$ & 14 \\
9 & $C_{3}^{2}$ & 3 \\
10 & $C_{10}$ & 1 \\
12 & $C_{12}$ & 3 \\
12 & $D_{12}$ & 8 \\
12 & $C_{6} \times C_{2}$ & 1 \\
16 & $C_{2} .\left(C_{4} \times C_{2}\right)$ & 1 \\
16 & $D_{8} \times C_{2}$ & 3 \\
18 & $C_{6} \times C_{3}$ & 1 \\
24 & $C_{12} \times C_{2}$ & 1 \\
24 & $S L(2,3)$ & 1 \\
\hline
\end{tabular}

TABLE 1. Holonomy groups of infra-nilmanifolds modeled on Heis 5 .

The first four groups in this list are split extensions of the form $F \ltimes \mathbb{Z}^{4}$, where $F$ is a nonsolvable finite group. Hence, these groups contain a noncyclic finite subgroup and therefore, they cannot be an underlying crystallographic group of the desired type.

The other two groups arise as an extension of the form $1 \rightarrow \mathbb{Z}^{4} \rightarrow Q \rightarrow A_{5} \rightarrow 1$, which is presented by a cohomology class of $H^{2}\left(A_{5}, \mathbb{Z}^{4}\right) \cong \mathbb{Z}_{3}$ (see [Brown et al. 78]). However, $A_{5}$ contains a noncyclic subgroup $V \cong C_{2}^{2}$ of order 4. As $H^{2}\left(V, \mathbb{Z}^{4}\right)$ is a 2-group, the restriction map res: $H^{2}\left(A_{5}, \mathbb{Z}^{4}\right) \rightarrow H^{2}\left(V, \mathbb{Z}^{4}\right)$ must be the zero map. This shows that the group $V$ lifts to a subgroup of $Q$, showing that $Q$ contains a noncyclic finite subgroup and hence can be disregarded in our search for the possible underlying crystallographic groups.

Then, we compute that 199 of the oriented and solvable groups have only cyclic finite subgroups. Finally, we use the algorithms of Section 3 to determine that 135 of the 199 groups have an extension $G$ which is torsion-free and yields that $Z(\operatorname{Fitt}(G)) \cong \mathbb{Z}$. Note that by Theorem 3.8, these two conditions ("torsion-free" and " $Z(\operatorname{Fitt}(G)) \cong \mathbb{Z}$ ") can be tested separately. In turn, this can be done by Theorems 3.3 and 3.6. The remaining 
135 groups arise as underlying crystallographic groups of an almost Bieberbach group corresponding to an infranilmanifold modeled on a 5-dimensional Heisenberg Lie group. Their holonomy groups are listed in Table 1, together with the number of crystallographic groups having this holonomy and lifting to an almost Bieberbach group of the desired type.

Finally, we note that the overall computation takes roughly 35 minutes on an average $\mathrm{PC}$, where most of this time is spent in computing the finite subgroup classes of the considered crystallographic groups.

Note that the same kind of computations can now also be used to check the main result of [Lee 00] in a few minutes.

\section{APPENDIX A}

In Section 5.1 we described an algorithm to determine the almost Bieberbach groups $G$ whose Fitting subgroup $N$ has Hirsch length 4 and class 2. Table 2 presents a summary of the results obtained by this algorithm; the information has been reduced to fit the table. For more details, such as explicit descriptions of the desired extensions, we suggest using our publically available implementations. We note that it takes only about three minutes to determine the information described in the algorithm "AlmostBieberbachExtensions" from Section 5.1 for all 3-dimensional crystallographic groups.

In the algorithm "AlmostBieberbachExtension" from Section 5.1, we consider a 3 -dimensional crystallographic group $Q$ and check that it has no noncyclic finite subgroup. Then we determine the relevant $Q$-modules $Z \cong \mathbb{Z}$ and construct $H^{2}(Q, Z)$. It remains to determine the subset $T \subset H^{2}(Q, Z)$ of elements of infinite order and the subset $S \subset H^{2}(Q, Z)$ of elements corresponding to extensions which are not torsion-free.

Table 2 lists all 3-dimensional crystallographic groups which have an almost Bieberbach extension of the desired type. It indicates the type of module of this extension, and it displays the isomorphism type of $H^{2}(Q, Z)$. The subset $T$ can be read directly from the abelian invariants of $H^{2}(Q, Z)$. The subset $S$ is determined as a union $S=\cup_{i=1}^{s} S_{i}$ where each $S_{i}$ corresponds to a representative of the finite subgroups of prime order $H_{1}, \ldots, H_{s}$ in $Q$. The table lists the orders of these subgroups $H_{1}, \ldots, H_{s}$.

The first entry in the table is the number of the crystallographic group as it appears in the International Tables for Crystallography ([Hahn 87]). They are included here for ease of comparing our table with the tables in [Dekimpe 96].
Finally, comparing Table 2 to the classification of the almost Bieberbach groups in [Dekimpe 96], we note that both determinations coincide except for the almost Bieberbach extensions of the group $Q$ of type 143 which are missing from [Dekimpe 96].

Thus, we include an explicit outline of these extensions here. The notation follows the notation in [Dekimpe 96] and we refer to [Dekimpe 96] for more information.

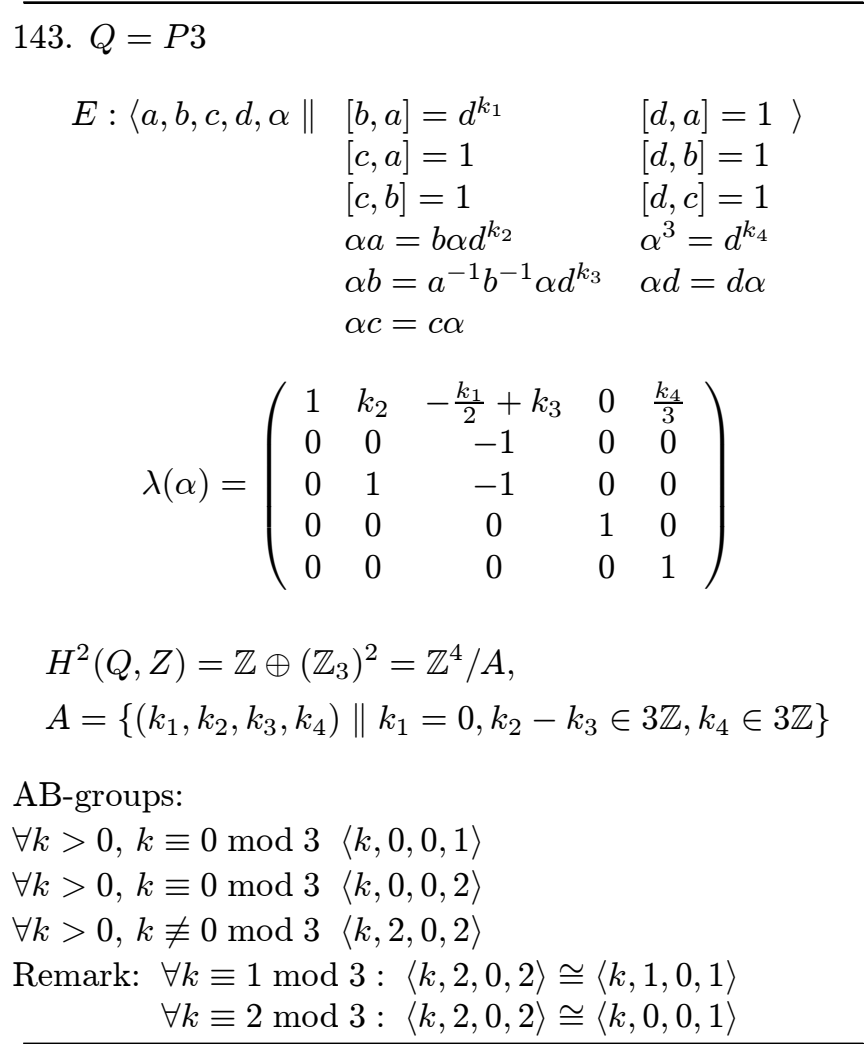

The line "143. $Q=P 3$ " indicates that we are dealing with the crystallographic group $Q$ with number 143 in [Hahn 87]. This is, in fact, the group of type $5 / 1 / 2 / 01$ in [Brown et al. 78]. Below that, one finds parameterized presentations for all extensions $E$ of $\mathbb{Z}$ by this crystallographic group $Q$ (trivial action). Note that these presentations are not polycyclic presentations (but they are almost). The matrix $\lambda(\alpha)$ describes a matrix representation for each of the extensions $E$. The images of the other generators of $E$ are described on page 168 of [Dekimpe 96]. Thereafter, a description of $H^{2}(Q, \mathbb{Z})$ is given in terms of the parameters appearing in the presentation of $E$. In fact, this is completely comparable with the situation described in Section 2.3.2. Finally, which parameters correspond to torsion-free extensions (almost Bieberbach groups) are indicated; information about isomorphisms between torsion-free extensions is also given. 


\begin{tabular}{|c|c|c|c|c|}
\hline \multicolumn{3}{|c|}{ Crystallographic Group $Q$} & \multirow{2}{*}{ Module $Z \cong \mathbb{Z}$} & \multirow[t]{2}{*}{ Type of $H^{2}(Q, Z)$} \\
\hline IT & Type & Orders of $H_{1}, \ldots, H_{s}$ & & \\
\hline 1 & $(1,1,1,1)$ & () & trivial & $(0,0,0)$ \\
\hline 2 & $(1,2,1,1)$ & $(2,2,2,2,2,2,2,2)$ & trivial & $(2,2,2,2,0,0,0)$ \\
\hline 3 & $(2,1,1,1)$ & $(2,2,2,2)$ & trivial & $(2,2,2,0)$ \\
\hline 4 & $(2,1,1,2)$ & () & trivial & $(2,2,0)$ \\
\hline 4 & $(2,1,1,2)$ & () & nontrivial & $(0,0)$ \\
\hline 5 & $(2,1,2,1)$ & $(2,2)$ & trivial & $(2,2,0)$ \\
\hline 6 & $(2,2,1,1)$ & $(2,2)$ & trivial & $(2,2,0)$ \\
\hline 7 & $(2,2,1,2)$ & () & trivial & $(2,0)$ \\
\hline 7 & $(2,2,1,2)$ & () & nontrivial & $(2,0,0)$ \\
\hline 8 & $(2,2,2,1)$ & (2) & trivial & $(2,0)$ \\
\hline 9 & $(2,2,2,2)$ & () & trivial & $(0)$ \\
\hline 9 & $(2,2,2,2)$ & () & nontrivial & $(0,0)$ \\
\hline 13 & $(2,3,1,2)$ & $(2,2,2,2,2,2)$ & trivial & $(2,2,2,2,0)$ \\
\hline 11 & $(2,3,1,3)$ & $(2,2,2,2,2)$ & trivial & $(2,2,2,2,0)$ \\
\hline 14 & $(2,3,1,4)$ & $(2,2,2,2)$ & trivial & $(2,2,4,0)$ \\
\hline 14 & $(2,3,1,4)$ & $(2,2,2,2)$ & nontrivial & $(2,2,0,0)$ \\
\hline 15 & $(2,3,2,2)$ & $(2,2,2,2,2)$ & trivial & $(2,2,2,0)$ \\
\hline 18 & $(3,1,1,3)$ & $(2,2)$ & nontrivial & $(2,0)$ \\
\hline 19 & $(3,1,1,4)$ & () & nontrivial & (0) \\
\hline 19 & $(3,1,1,4)$ & () & nontrivial & (0) \\
\hline 19 & $(3,1,1,4)$ & () & nontrivial & (0) \\
\hline 27 & $(3,2,1,3)$ & $(2,2,2,2)$ & nontrivial & $(2,2,2,0)$ \\
\hline 30 & $(3,2,1,4)$ & $(2,2)$ & nontrivial & $(2,2,0)$ \\
\hline 32 & $(3,2,1,5)$ & $(2,2)$ & nontrivial & $(2,2,0)$ \\
\hline 34 & $(3,2,1,6)$ & $(2,2)$ & nontrivial & $(2,2,0)$ \\
\hline 26 & $(3,2,1,7)$ & $(2,2)$ & nontrivial & $(2,2,0)$ \\
\hline 31 & $(3,2,1,8)$ & $(2)$ & nontrivial & $(2,0)$ \\
\hline 29 & $(3,2,1,9)$ & () & nontrivial & $(2,0)$ \\
\hline 29 & $(3,2,1,9)$ & () & nontrivial & $(2,2,0)$ \\
\hline 29 & $(3,2,1,9)$ & () & nontrivial & $(2,0)$ \\
\hline 33 & $(3,2,1,10)$ & () & nontrivial & $(0)$ \\
\hline 33 & $(3,2,1,10)$ & () & nontrivial & $(4,0)$ \\
\hline 33 & $(3,2,1,10)$ & () & nontrivial & (0) \\
\hline 37 & $(3,2,2,2)$ & $(2,2,2)$ & nontrivial & $(2,2,0)$ \\
\hline 36 & $(3,2,2,3)$ & $(2)$ & nontrivial & $(2,0)$ \\
\hline 41 & $(3,2,3,4)$ & (2) & nontrivial & $(2,2,0)$ \\
\hline 43 & $(3,2,4,2)$ & (2) & nontrivial & $(2,0)$ \\
\hline 45 & $(3,2,5,3)$ & $(2,2)$ & nontrivial & $(2,2,0)$ \\
\hline 60 & $(3,3,1,10)$ & $(2,2,2)$ & nontrivial & $(2,2,0)$ \\
\hline 56 & $(3,3,1,12)$ & $(2,2,2,2)$ & nontrivial & $(2,2,0)$ \\
\hline 62 & $(3,3,1,15)$ & $(2,2,2)$ & nontrivial & $(2,2,0)$ \\
\hline 61 & $(3,3,1,16)$ & $(2,2)$ & nontrivial & $(2,2,0)$ \\
\hline 61 & $(3,3,1,16)$ & $(2,2)$ & nontrivial & $(2,2,0)$ \\
\hline 61 & $(3,3,1,16)$ & $(2,2)$ & nontrivial & $(2,2,0)$ \\
\hline 75 & $(4,1,1,1)$ & $(2,2,2)$ & trivial & $(2,4,0)$ \\
\hline 76 & $(4,1,1,2)$ & () & trivial & $(2,0)$ \\
\hline 77 & $(4,1,1,3)$ & $(2,2,2)$ & trivial & $(2,2,0)$ \\
\hline 79 & $(4,1,2,1)$ & $(2,2)$ & trivial & $(4,0)$ \\
\hline 80 & $(4,1,2,2)$ & $(2)$ & trivial & $(2,0)$ \\
\hline 81 & $(4,2,1,1)$ & $(2,2,2)$ & trivial & $(2,2,4,0)$ \\
\hline 82 & $(4,2,2,1)$ & $(2,2)$ & trivial & $(4,4,0)$ \\
\hline 85 & $(4,3,1,3)$ & $(2,2,2,2)$ & trivial & $(2,2,4,0)$ \\
\hline 86 & $(4,3,1,4)$ & $(2,2,2,2)$ & trivial & $(2,2,4,0)$ \\
\hline 88 & $(4,3,2,2)$ & $(2,2,2)$ & trivial & $(2,4,0)$ \\
\hline 103 & $(4,5,1,3)$ & $(2,2,2)$ & nontrivial & $(2,4,0)$ \\
\hline 106 & $(4,5,1,6)$ & $(2,2)$ & nontrivial & $(4,0)$ \\
\hline 104 & $(4,5,1,7)$ & $(2,2)$ & nontrivial & $(4,0)$ \\
\hline 110 & $(4,5,2,4)$ & (2) & nontrivial & $(4,0)$ \\
\hline
\end{tabular}

TABLE 2. Determining the 4-dimensional almost Bieberbach groups. 


\begin{tabular}{|c|c|c|c|c|}
\hline \multicolumn{3}{|c|}{ Crystallographic Group $Q$} & \multirow[t]{2}{*}{ Module $Z \cong \mathbb{Z}$} & \multirow[t]{2}{*}{ Type of $H^{2}(Q, Z)$} \\
\hline IT & Type & Orders of $H_{1}, \ldots, H_{s}$ & & \\
\hline 114 & $(4,6,1,4)$ & $(2,2)$ & nontrivial & $(4,0)$ \\
\hline 146 & $(5,1,1,1)$ & (3) & trivial & $(3,0)$ \\
\hline 143 & $(5,1,2,1)$ & $(3,3,3)$ & trivial & $(3,3,0)$ \\
\hline 144 & $(5,1,2,2)$ & () & trivial & $(3,0)$ \\
\hline 148 & $(5,2,1,1)$ & $(2,2,2,2,3)$ & trivial & $(2,6,0)$ \\
\hline 147 & $(5,2,2,1)$ & $(2,2,2,2,3,3)$ & trivial & $(2,6,0)$ \\
\hline 161 & $(5,4,1,2)$ & (3) & nontrivial & $(3,0)$ \\
\hline 158 & $(5,4,2,2)$ & $(3,3,3)$ & nontrivial & $(3,3,0)$ \\
\hline 159 & $(5,4,3,2)$ & $(3,3)$ & nontrivial & $(3,0)$ \\
\hline 168 & $(6,1,1,1)$ & $(2,2,3,3)$ & trivial & $(6,0)$ \\
\hline 172 & $(6,1,1,2)$ & $(2,2)$ & trivial & $(2,0)$ \\
\hline 173 & $(6,1,1,3)$ & $(3,3)$ & trivial & $(3,0)$ \\
\hline 169 & $(6,1,1,4)$ & () & trivial & $(0)$ \\
\hline 174 & $(6,2,1,1)$ & $(2,2,3,3,3)$ & trivial & $(6,6,0)$ \\
\hline 176 & $(6,3,1,2)$ & $(2,2,2,3,3)$ & trivial & $(2,6,0)$ \\
\hline 184 & $(6,5,1,2)$ & $(3,3,2,2)$ & nontrivial & $(6,0)$ \\
\hline
\end{tabular}

TABLE 2 (continued).

\section{REFERENCES}

[Brown et al. 78] H.Brown, R. Bülow, J. Neubüser, H. Wondratscheck, and H. Zassenhaus. Crystallographic groups of four-dimensional Space. Wiley, New York, 1978.

[Brown 82] K. S. Brown. Cohomology of groups, volume 87 of Grad. Texts in Math. Springer-Verlag New York Inc., New York, 1982.

[Conner and Raymond 71] P. E. Conner, and F. Raymond. "Actions of compact Lie groups on aspherical manifolds." in Topology of Manifolds, Proc. Univ. Georgia 1969, pp. 227-264, 1971.

[Conner and Raymond 77] P. E. Conner, and F. Raymond. "Deforming Homotopy Equivalences to Homeomorphisms in Aspherical Manifolds." Bull. A.M.S., 83:1 (1977) 36-85.

[Dekimpe 96] K. Dekimpe. Almost-Bieberbach Groups: Affine and Polynomial Structures, volume 1639 of Lect. Notes in Math. Springer-Verlag, Berlin-Heidelberg, 1996.

[Dekimpe and Eick 01a] K. Dekimpe, and B. Eick. Aclib $A$ GAP 4 package, 2001. See [Gap 00].

[Dekimpe and Eick 01b] K. Dekimpe, and B. Eick. "Computations with almost-crystallographic groups." Proceedings of Groups, St. Andrews 2001, edited by Campbell and Robertson, 2001.

[Dekimpe and Igodt 97] K. Dekimpe, and P. Igodt. "Polycyclic-by-finite groups admit a bounded-degree polynomial structure." Invent. Math. 129:1 (1997), 121-140.
[Dekimpe et al. 95] K. Dekimpe, P. Igodt, S. Kim, and K. B. Lee. "Affine structures for closed 3-dimensional manifolds with NIL-geometry." Quart. J. Math. 46:2 (1995), 141-167.

[Eick 01a] B. Eick. "Computing with infinite polycyclic groups." in Proceedings Groups and Computation III, edited by Seress and Kantor, pp. 139-153, de Gruyter, Berlin, 2001.

[Eick 01b] B. Eick. Algorithms for polycyclic groups. Habilitationsschrift, Universität Kassel, 2001.

[Eick and Nickel 00] B. Eick, amd W. Nickel. Polycyclic - A GAP 4 package, 2000. See [Gap 00].

[Gap 00] The GAP Group, GAP-Groups, Algorithms and Programming, Version 4.2, 2000. (http://www.gapsystem.org).

[Goze and Khkimdjanov 96] M. Goze and Y. Khkimdjanov. "Nilpotent Lie Algebras." in Mathematics and Its Applications, Kluwer Academic Publishers, Norwood, MA, 1996.

[Hahn 87] T. Hahn. International tables for crystallography, A. Space-group symmetry, (International union of crystallography) Reidel, Dordrecht, 1987.

[Lee 88] K. B. Lee. "There are only finitely many infranilmanifolds under each nilmanifold." Quart. J. Math. 39:2 (1988), 61-66.

[Lee 00] Lee, K. B. "Infranil-manifolds modeled on Heis 5 ," Preprint, 2000. 
[Lee and Szczepański 00] K. B Lee, and A. Szczepański. "Maximal Holonomy of Almost Bieberbach Groups For Heis $5 . "$ Geom. Dedicata 87:1-3 (2000), 167-180.

[Robinson 82] D. J. S. Robinson.. A course in group theory. Springer-Verlag, New York, 1982.
[Segal 83] D. Segal, D. Polycyclic Groups, Cambridge University Press, Cambridge, UK, 1983.

[Sims 94] C. C. Sims. Computation with finitely presented groups, Cambridge University Press, Cambridge, UK, 1994.

Karel Dekimpe, Katholieke Universiteit Leuven, Campus Kortrijk, B-8500 Kortrijk, Belgium

(Karel.Dekimpe@kulak.ac.be)

Bettina Eick, Universität Braunschweig, Institut für Geometrie, D-38106 Braunschweig, Germany (beick@tu-bs.de)

Received June 25, 2001; accepted in revised form October 23, 2001. 